\title{
1 A selective bottleneck during host entry drives the evolution of new legume symbionts
}

2 Ginaini Grazielli Doin de Moura ${ }^{1}$, Saida Mouffok ${ }^{1}$, Nil Gaudu ${ }^{1}$, Anne-Claire Cazalé ${ }^{1}$, Marine Milhes ${ }^{2}$,

3 Tabatha Bulach², Sophie Valière ${ }^{2}$, David Roche ${ }^{3}$, Jean-Baptiste Ferdy ${ }^{4}$, Catherine Masson-Boivin ${ }^{1}$,

4 Delphine Capela ${ }^{1}$ and Philippe Remigi ${ }^{1}$

5

6

7

8

${ }^{1}$ LIPME, Université de Toulouse, INRAE, CNRS, Castanet-Tolosan, France

${ }^{2}$ INRAE, US1426, GeT-PlaGe, Genotoul, Castanet-Tolosan, France

${ }^{3}$ Génomique Métabolique, Genoscope, Institut François Jacob, CEA, CNRS, Univ Evry, Université ParisSaclay, 91057 Evry, France

${ }^{4}$ CNRS, UMR5174 EDB, Université Toulouse 3 Paul Sabatier, Toulouse, France.

Email for correspondence: delphine.capela@inrae.fr; philippe.remigi@inrae.fr

\section{Abstract}

During the emergence of new host-microbe symbioses, multiple selective pressures -acting at the different steps of the microbial life cycle- will shape the phenotypic traits that jointly determine microbial fitness. However, the relative contribution of these different selective pressures on the adaptive trajectories of microbial symbionts are still poorly known. Here we characterized the dynamics of phenotypic adaptation and the underlying genetic bases during the experimental evolution of a plant pathogenic bacterium into a legume symbiont. We show that fast adaptation was supported by the sequential fixation of mutational cohorts within populations, and dominated by selection for host entry competitiveness over within-host proliferation. Computer simulations predict that this effect emerges from the presence of a strong selective bottleneck at host entry before bacterial multiplication. However, identifying and characterizing adaptive mutations revealed recurrent couplings between the two phenotypic traits, further increasing the efficiency of hostmediated selection of new symbionts.

\section{Main}

Many bacterial lineages have evolved the capacity to establish symbiotic associations, either beneficial, neutral or parasitic, with eukaryotic hosts. These interactions form a dynamic continuum along which bacteria can move ${ }^{1}$. Lifestyle changes may arise due to ecological (resource availability, host environment changes or host shifts) or genomic (mutations, acquisition of new genetic material) modifications. These new interactions are often initially sub-optimal for the bacterial partner-4, which 
may then adapt to the selective pressures associated with its new life-cycle. When the microbes are transmitted horizontally, they must alternate between at least two different habitats, the host and the environment, which will impose a variety of constraints and selective pressures ${ }^{5,6}$. Biphasic life cycles therefore require bacteria to be able to enter and exit their hosts, and replicate and persist within each habitat. Surviving and persisting in different niches requires adapting to abiotic stressors, host immunity, competitors, as well as being able to use specific nutrient sources. However, the relative influence of the different selective pressures on the dynamics of bacterial adaptation to a new interaction and on the evolution of phenotypic traits is poorly known.

Rhizobia are examples of facultative host-associated bacteria that can either live freely in soil or in symbiotic mutualistic associations with legume plants ${ }^{7}$. In most legumes, rhizobia penetrate the root tissue through the formation of so-called infection threads (ITs). Most of the time, only one bacterium attaches to the root hair and initiates the formation of $1 \mathrm{Ts}^{8}$, thus creating a strong population bottleneck between the rhizosphere and internal root tissues. Bacteria then divide clonally within ITs, while at the same time a nodule starts to develop at the basis of the infected root hair. Hundreds of millions of bacteria are then released from ITs inside the cells of the developing nodule and differentiate into nitrogen fixing bacteroids ${ }^{9,10}$. After several months, in nature, nodule senescence leads to the release of a part of bacterial nodule population in the surrounding soil. To fulfil their life cycle, rhizobia rely on the activity of numerous bacterial genes, allowing signal exchanges with the host plant, proliferation and metabolic exchanges within nodule cells, while maintaining free-living proficiency ${ }^{7}$. Somewhat surprisingly given their complex and specialized lifestyle, rhizobia evolved several times independently. Horizontal transfer of key symbiotic genes is a necessary, though often not sufficient, condition for a new rhizobium to emerge ${ }^{11-14}$. Additional steps of adaptation of the recipient bacterium, occurring during evolution under plant selection, are believed to be needed to actualize the symbiotic potential of emerging rhizobia ${ }^{15}$.

In a previous study, we used experimental evolution to convert the plant pathogen Ralstonia solanacearum into an intracellular legume symbiont ${ }^{16}$. Here, we analysed the dynamics of molecular evolution in 5 lineages evolved for 35 cycles of nodulation through whole-population genome sequencing and examined the selective and genetic bases of adaptation. Adaptation proceeded rapidly during the first cycles of evolution, and was underpinned by the fixation of successive cohorts of mutations within populations. We then identified adaptive mutations in two lineages and evaluated their effect on each symbiotic stage of the rhizobial life cycle. Our experimental data indicated that selection for host entry outweighs the selection for multiplication within host. Computer simulations further showed that the selective nodulation bottleneck and the chronology of symbiotic events are critical drivers of this evolutionary pattern. 


\section{Results}

\section{Fast adaptation of new legume symbionts during the first cycles of evolution}

To investigate the genetic and evolutionary conditions that promote the evolution of new rhizobia, we previously transferred a symbiotic plasmid (pRalta) from the rhizobium Cupriavidus taiwanensis LMG19424 into the plant pathogen Ralstonia solanacearum GMI1000. We obtained three nodulating ancestors, two infecting nodules intracellularly (CBM212, CBM349) and one infecting nodules extracellularly $(\mathrm{CBM} 356)^{14}$, that we evolved in 18 parallel bacterial lineages for 16 serial cycles of nodulation on Mimosa pudica plants ${ }^{16}$. Here we continued the evolution of five of these lineages (referred to as lineages B, F, G, K, and M) until cycle 35 (Fig. 1a, Extended Data Fig. 1, Supplementary Table 1). After 35 cycles, no nitrogen fixation sustaining plant growth was observed. Therefore, we focus exclusively on the analysis of changes in bacterial fitness in this manuscript. To analyse the dynamics of fitness changes over time, we compared the relative fitness of the nodulating ancestors and evolved clones from cycles 16 and 35 by replaying one nodulation cycle in competition with the natural symbiont $C$. taiwanensis. Fitness trajectories were similar in the five lineages. While the three nodulating ancestors were on average $10^{6}$ times less fit than $C$. taiwanensis, fitness improved very quickly during the first 16 cycles, then slower until cycle 35 (Fig. 1b,c). On average, evolved clones isolated at cycle 16 and cycle 35 were 47 and 19 times less fit than C. taiwanensis, respectively (Fig. 1c, and Supplementary Table 2).

Because bacterial fitness in our system depends on both the capacity of strains to enter the host and induce nodule formation (nodulation competitiveness) and to multiply within these nodules (withinhost proliferation), we analysed how each of these two fitness components changed during the experiment (Fig. 1b,c,d). In the five lineages, evolutionary trajectories of nodulation competitiveness resembled that of fitness with fast improvement during the first 16 cycles, and a slow-down during the last cycles. On average, nodulating ancestors were $5 \times 10^{4}$ times less competitive than $C$. taiwanensis, while cycle 16 and cycle 35 evolved clones were only 17 and 4 times less competitive than $C$. taiwanensis, respectively (Fig. 1c and Supplementary Table 2). Moreover, evolved clones B16, B35, G16, G35 and M35 were not statistically different from C. taiwanensis in terms of nodulation competitiveness. Within-host proliferation also improved mostly during the first 16 cycles in all lineages. The rate of improvement then slowed down in two lineages and continued to increase significantly in the three others. As previously published, the three nodulating ancestors display different capacities to infect nodules ${ }^{14,16}$, the extracellularly infective ancestor CBM356 being the less infective (750 times less than C. taiwanensis) and the intracellularly infective CBM349 being the most 
bioRxiv preprint doi: https:/doi.org/10.1101/2022.03.03 482760; this version posted March 4, 2022. The copyright holder for this preprint (which was not certified by peer review) is the author/funder, who has granted bioRxiv a license to display the preprint in perpetuity. It is made available under aCC-BY-NC 4.0 International license. infective (45 times less than C. taiwanensis). On average, cycle 16 and cycle 35 evolved clones were 8 and 5 times less infective than C. taiwanensis (Fig. 1d and Supplementary Table 2). Altogether, the symbiotic properties of evolved clones improved rapidly during the first cycles of the evolution experiment and then adaptation slowed down. Both symbiotic traits, nodulation competitiveness (i.e. host entry) and within-host proliferation, improved greatly. However, gains in nodulation competitiveness (average factor of 11,500 between ancestors and cycle 35 clones) were ca. 150 times higher than gains in proliferation (average factor of 74 between ancestors and cycle 35 clones). Moreover, although the difference in nodulation competitiveness between $C$. taiwanensis and the nodulating ancestors was much more important $\left(>10^{4}\right.$ fold) than the difference in proliferation $\left(<10^{3}\right.$ fold $)$, nodulation competitiveness of evolved clones reached the level of $C$. taiwanensis in three lineages ( $B, G$ and $M$ ) while within host proliferation remained lower than $C$. taiwanensis in all of them after 35 cycles.

a Experimental setup and phenotypic tests

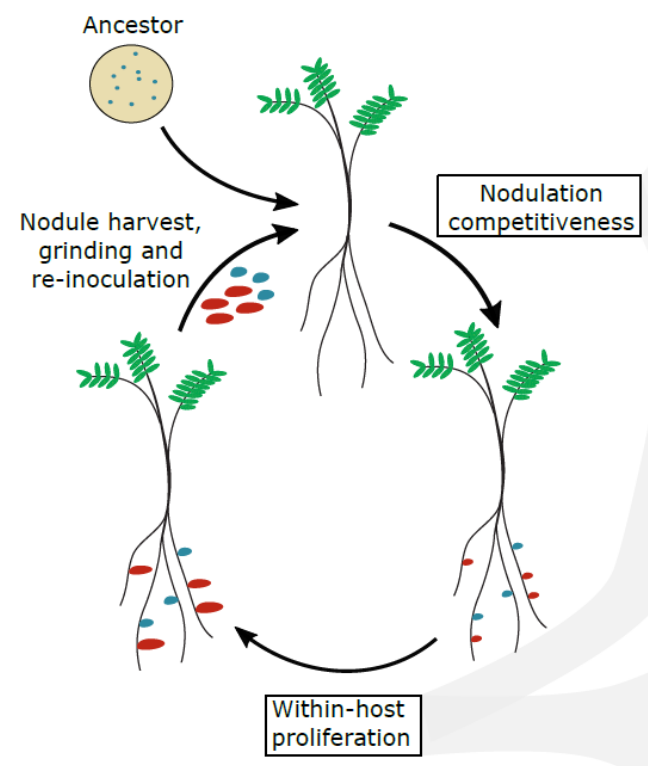

118
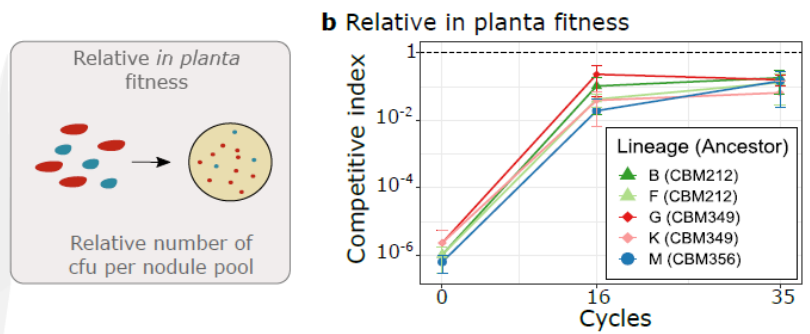

c Nodulation competitiveness
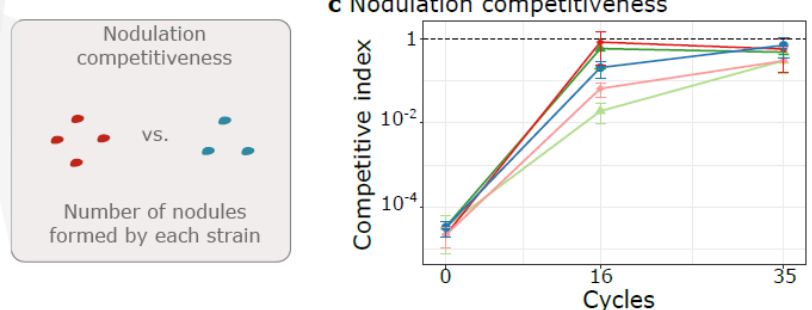

d Within-host proliferation
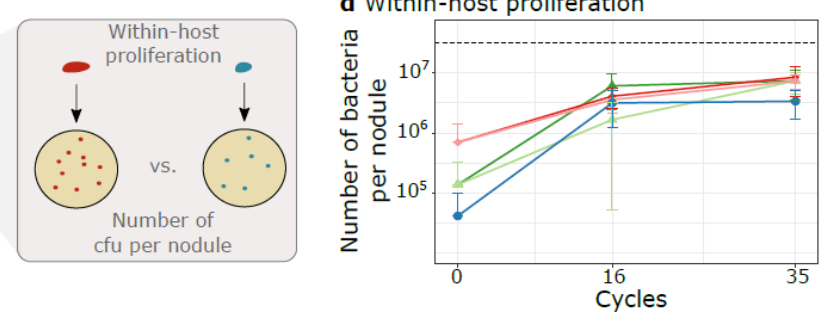

Fig. 1 Evolution of the symbiotic properties of Ralstonia clones along evolution cycles.

a, Left: overview of the evolution cycles showing the symbiotic steps determining bacterial fitness (nodulation competitiveness and within-host proliferation) and human intervention (nodule harvest, grinding and re-inoculation).

Nodule colors reflect bacterial genotypes: blue represents the ancestor, and red represents a mutant with increased fitness. Right: schematic representation of the main phenotypic measurements performed in this work, where the symbiotic phenotypes of one evolved strain can be compared to those of a reference strain (e.g. the reference symbiont C. taiwanensis). Competitive indexes for in planta fitness are calculated as the ratio of evolved vs. reference clones in nodule bacterial populations normalized by the ratio of strains in the inoculum. Competitive indexes for nodulation 

proliferation is measured in independent single inoculations of each strain. b-d, Relative in planta fitness (b), nodulation competitiveness (c) and within-host proliferation (d) of nodulating ancestors (cycle 0 ) and evolved clones isolated from cycles 16 and 35 were compared to $C$. taiwanensis. Values correspond to means \pm standard deviations. Data were obtained from at least three independent experiments. For each experiment, nodules were harvested from 10 plants (b), 20 plants (c) and 6 plants (d). The sample size $(n)$ is equal to $n=3(b, c)$ or comprised between $n=15-18$ (d). Raw data are available in Supplementary Table 2. cfu: colony-forming units.

The dynamics of molecular evolution is characterized by multiple selective sweeps of large mutational cohorts

The rapid adaptation in this experiment was striking given that strong nodulation bottlenecks (limiting effective population size at each cycle (Supplementary Table 1)) are generally known to limit the rate of adaptive evolution ${ }^{17}$. To describe the dynamics of evolution at the molecular level, we performed whole-population sequencing of evolved lineages using the Illumina sequencing technology. Populations of the five lineages were sequenced every other cycle until cycle 35 with a minimum sequencing coverage of 100x and a median of 347x (Supplementary Table 3). We detected a very large number of mutations in all sequenced populations (a total of 4114 mutations, between 382 and 1204 mutations per lineage with 23.6 new mutations per cycle on average) (Extended Data Table 1 and Supplementary Tables 4-8). This high number of mutations is a result of transient hypermutagenesis occurring in the rhizospher ${ }^{18}$. Mutations accumulated in the populations throughout the experiment, showing no sign of slowing down until the end of the experiment, which suggests that no anti-mutator mutations established in these populations as we might have expected ${ }^{19}$. We evaluated genetic parallelism among mutations detected above a frequency of $5 \%$ in the populations by computing $G$ scores $^{20}$, a statistics used to point out genes that could be mutated more often than expected by chance (Extended Data Table 2). In spite of high mutation rates that may obscure signals of genetic convergence, we detected signatures of parallelism at the gene level among our list of mutations (observed sum of $\mathrm{G}$ scores of 7,540.5, compared to a mean sum of 5,401.01 after 1,000 randomized simulations, $\left.Z=31.23, P<10^{-200}\right)$. The randomized simulations identified 171 genes with a higher number of mutations than expected by chance (Extended Data Table 2).

To simplify the analysis of mutational trajectories, we then focused on the 819 mutations (out of a total of 4414 detected mutations) that rose above a frequency of $30 \%$. We observed that groups of mutations showed correlated temporal trajectories and clustered them in cohorts ${ }^{21,22}$ (Fig. 2 and

159 Extended Data Table 3). Cohorts increased in frequency with variable speed, some being fixed within 1601 to 3 cycles (representing ca. 25-75 bacterial generations) while others reached fixation in up to 21 cycles (representing more than 500 bacterial generations). Fixed cohorts can be very large (up to 30 mutations) (Extended Data Table 3), which suggests hitchhiking of neutral or slightly deleterious 
bioRxiv preprint doi: https:/doi.org/10.1101/2022.03 03 482760; this version posted March 4, 2022. The copyright holder for this preprint (which was not certified by peer review) is the author/funder, who has granted bioRxiv a license to display the preprint in perpetuity. It is made available under aCC-BY-NC 4.0 International license.

mutations with one or several beneficial mutations acting as driver (or co-drivers) of the cohort ${ }^{21,23}$. Among the mutations that rose above $30 \%$ frequency at some stage of the experiment, $35 \%$ later declined until extinction. This is indicative of clonal interference, i.e. the co-occurrence of multiple subpopulations competing against each other within populations ${ }^{24}$, which can readily be observed on Muller plots for lineages B and G (Extended Data Fig. 2).

Overall, in these 5 lineages, the pattern of molecular evolution was characterized by a steady accumulation of mutations along the successive cycles and the formation of mutational cohorts, some of which containing a large number of mutations. Despite strong selective population bottlenecks at the root entry, mutation supply was not limiting as evidenced by the co-occurrence of competing adaptive mutations. Yet, multiple (and sometimes rapid) selective sweeps occurred throughout the 35 cycles in all lineages, indicating that strong selection is acting on these populations.
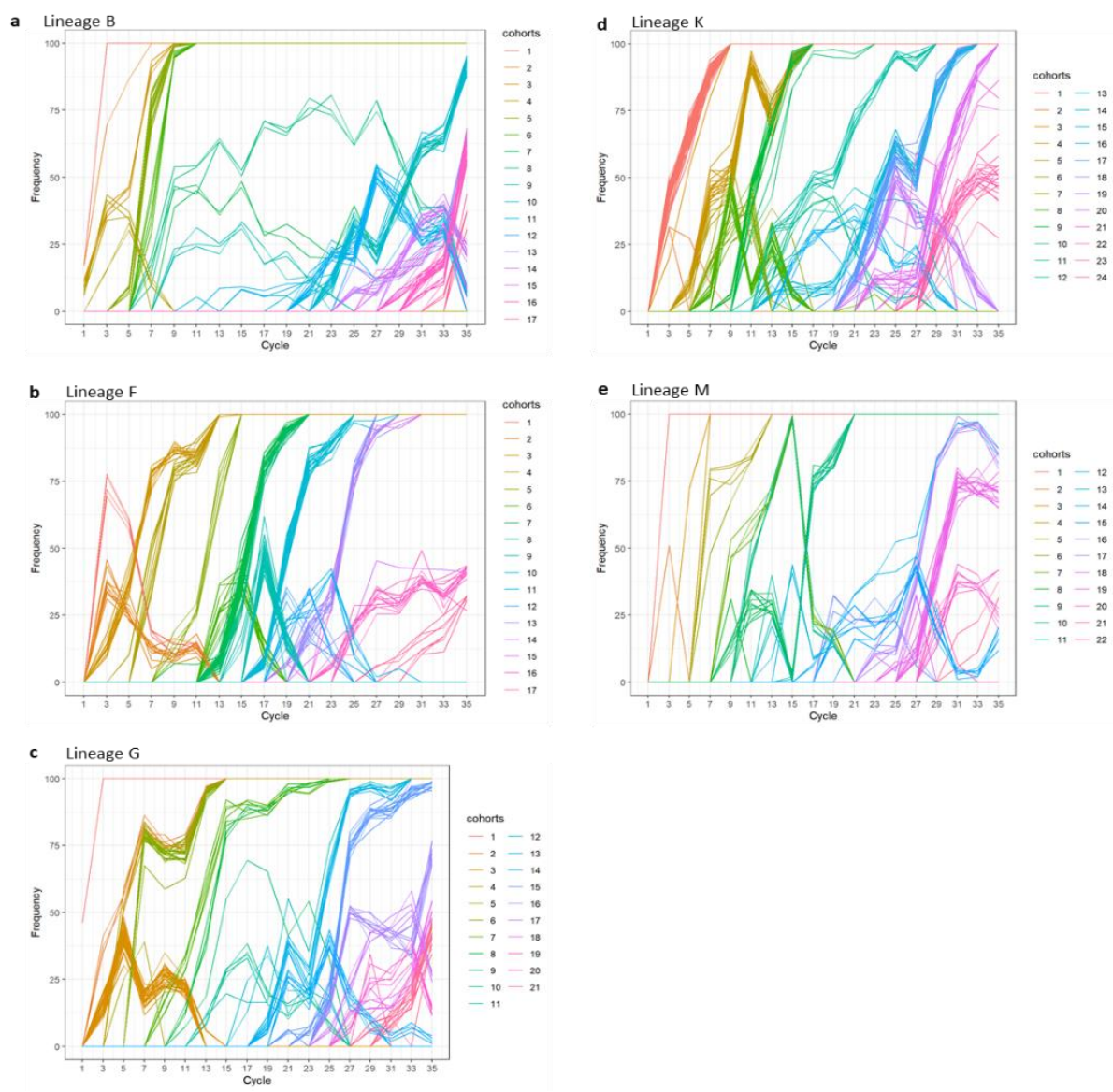

Fig. 2 Dynamics of molecular evolution

Allele frequency trajectories of mutations that attained a frequency of $30 \%$ in at least one population of the B, F, G, K and M lineages $(a, b, c, d, e)$. Mutations with similar trajectories were clustered in cohorts and represented by different colors. For simplicity, mutations travelling alone were also called cohorts. 
Next, we searched for driver mutations in fixed (or nearly fixed, $>90 \%$ frequency) cohorts from the two lineages that have the highest symbiotic fitness after 35 cycles: B and G. To do so, we identified, for each cohort of interest, a clone carrying all (or, when not available, almost all) previously fixed cohorts of mutations and used it to reconstruct individual point mutations from this cohort. This allowed us to test the fitness effect associated with each mutation in a relevant genetic background, taking into account possible epistatic effects arising from mutations that were previously acquired in this clone. Competitive index (ratio of strains carrying the mutant vs. the wild-type alleles in nodule populations normalized by the inoculum ratio) were measured for 44 mutations belonging to 14 cohorts from the two lineages (Fig. 3a,d and Supplementary Table 7). Twenty-eight mutations significantly increased the fitness of evolved clones and were thus beneficial for symbiosis while 11 mutations were neutral and 5 were slightly deleterious for symbiosis (Fig. 3a,d, Table 1 and Extended Data Table 4). Among the 44 genes targeted by the reconstructed mutations, 8 have a higher number of mutations than expected by chance (Extended Data Table 2) and adaptive mutations were identified in 6 of them.

Several interesting results emerge from this data set. First, some mutations show very strong adaptive effects, improving more than 100 times the fitness of bacteria, in particular during the first cycles of evolution. Second, many cohorts carry more than 1 adaptive mutation: six cohorts contain between 2 and 7 adaptive mutations. Third, $35 \%$ of adaptive mutations were found to be synonymous mutations, confirming that these mutations can play a significant role in adaptive evolution ${ }^{25}$. All adaptive synonymous mutations except one converted frequently used codons in $R$. solanacearum into unusual ones (Extended Data Table 5), which may explain their functional effects. Moreover, when inspecting gene annotations, we found that the 28 beneficial mutations target various biological functions (Table 1). Strikingly, nearly $40 \%$ affect regulatory functions such as global transcription regulators (efp $R$ and Rsc0965) ${ }^{26}$, putative quorum quenching (RSp0595), signal transduction (RSc1598), unknown transcription regulator (RSc0243), protein dephosphorylation (Rsp1469), protein folding (ppiB), degradosome (rhIE1), and DNA methylation (RSc1982). Another significant proportion of mutations (32\%) affect metabolism and transport genes ( $g l o A, p h b B$, RSc0194, RSc2277, Rsp1116, RSp1417, RSp1422, RSp1590 and pRALTA_0398) and 3 mutations are located in genes belonging to the type VI secretion system (T6SS) operon (tssJ, RSp0759, Rsp0769). The observed genetic architecture (i.e. the large number of genes and molecular functions targeted by adaptive mutations) indicates that symbiotic traits are complex and constitute a large mutational target for adaptive evolution. 
bioRxiv preprint doi: https://doi.org/10.1101/2022.03.03 482760; this version posted March 4, 2022. The copyright holder for this preprint (which was not certified by peer review) is the author/funder, who has granted bioRxiv a license to display the preprint in perpetuity. It is made available under aCC-BY-NC 4.0 International license.

a

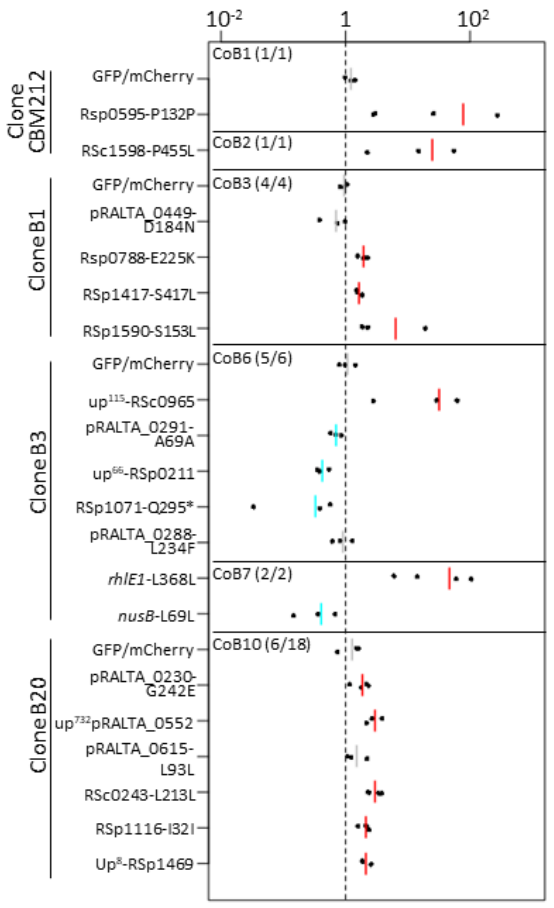

d

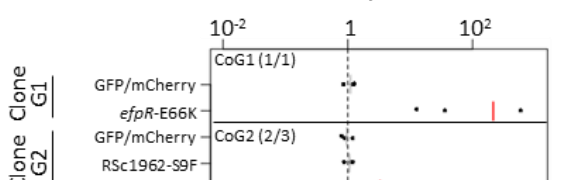

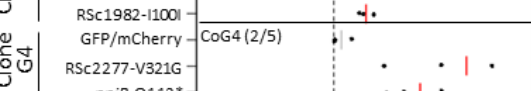

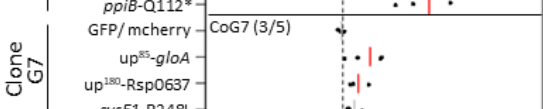

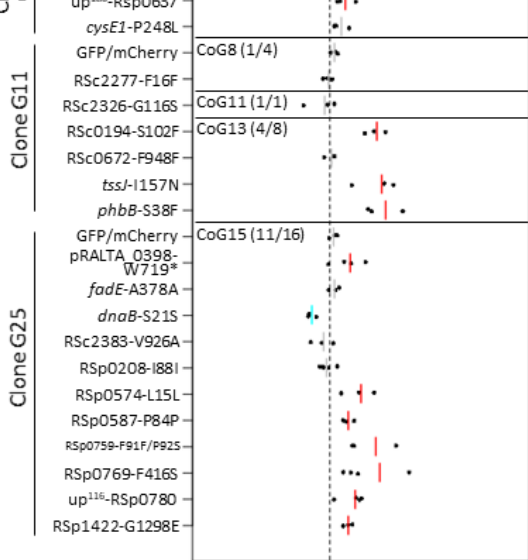

b

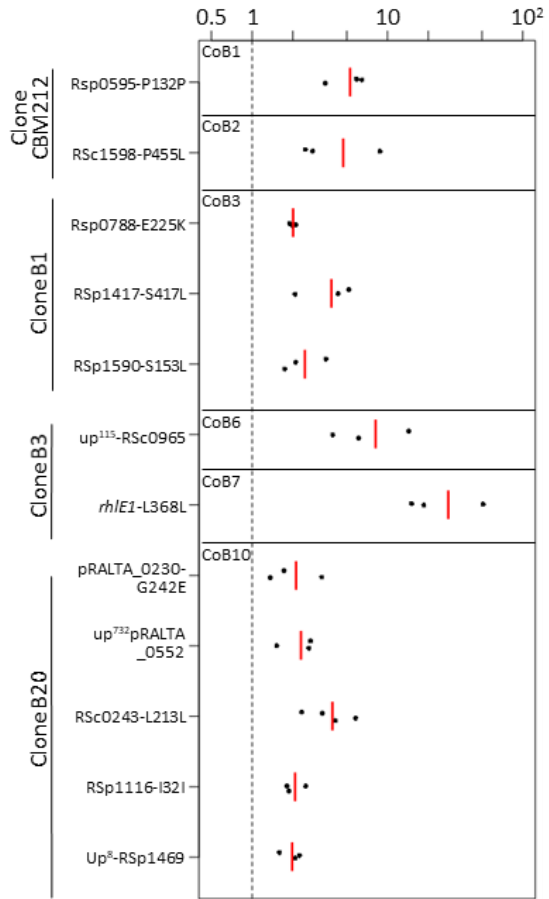

e

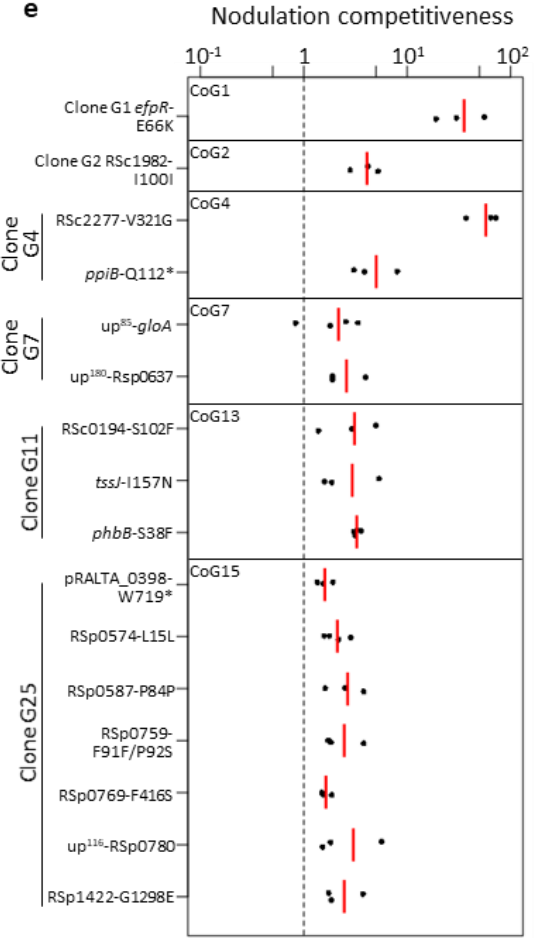

c

Number of bacteria per nodule

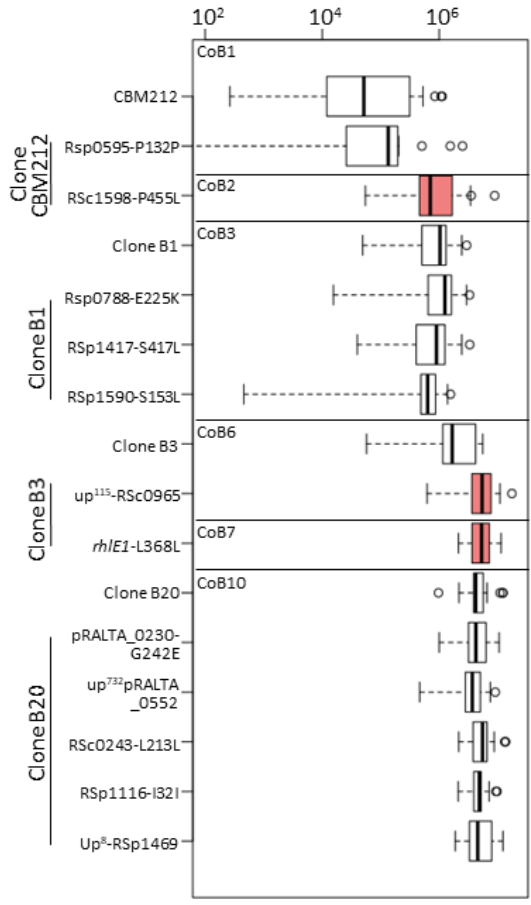

f

Number of bacteria per nodule

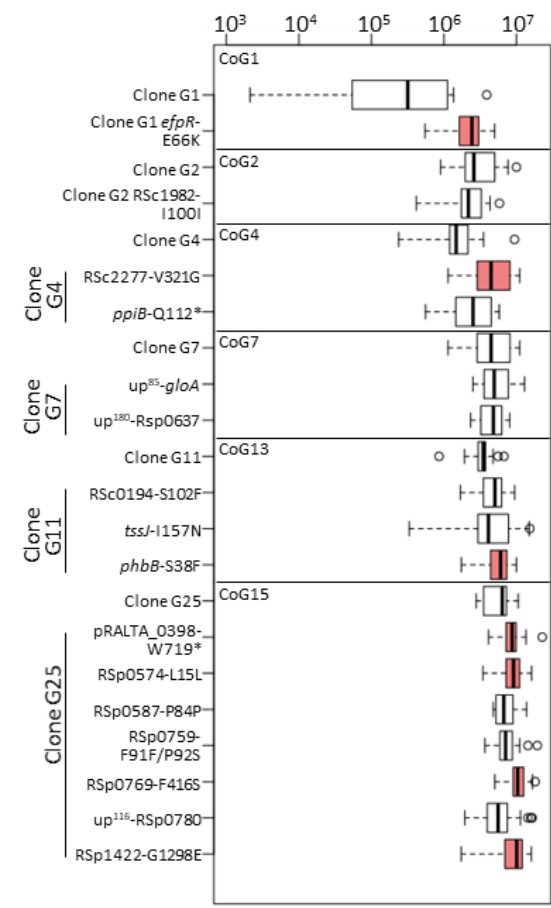

Fig. 3 Symbiotic phenotypes of reconstructed mutants and evolved clones from lineages B and G.

$\mathrm{a}, \mathrm{d}$, In planta relative fitness of evolved clones carrying reconstructed mutations from fixed mutational cohorts. Evolved clones in which mutations were reconstructed are indicated on the left of the graph. Horizontal lines separate the different mutational cohorts. Cohort number and the number of tested mutations from the cohort on the total number of mutations present in the cohort are indicated in brackets. Competitive indexes $(\mathrm{Cl})$ were calculated as the ratio of the mutant strain on the isogenic parental strain in bacterial nodule populations normalized by the inoculum ratio. GFP/mCherry correspond to control co-inoculation experiments of strains derived from the same evolved clone labelled with different fluorophore either GFP or mCherry. b,e, Nodulation competitiveness effect of adaptive mutants. Cl were calculated as the ratio of the number 
of nodules formed by the mutant strain on the number of nodules formed by the isogenic parental strain normalized by the inoculum ratio. $a, b, d, e$, vertical segments correspond to mean values of $\mathrm{Cl}$. Red segments indicate significantly beneficial mutations, gray segments indicate neutral mutations and blue segments indicate significantly deleterious mutations $(P<0.05$, t-test). c,f, Distribution of within-host proliferation values (number of bacteria per nodule) of adaptive mutants and the corresponding isogenic parental clones. Rectangles span the first quartiles to the third quartiles, bold segments inside the rectangle show the median, unfiled circles represent outliers, whiskers above and below each box show the minimum and the maximum in the absence of suspected outlying data. Red stars indicate the mutations that significantly improved infectivity compared to the parental evolved clone ( $P<0.05$, Wilcoxon test). a,b,c,d,e,f, Data were obtained from 3 to 5 independent experiments. For each experiment, nodules were harvested from 10 plants (a,d), 20 plants (b,e) and 6 plants $(c, f)$. The sample size $(n)$ is comprised between $n=3-5(a, d), n=3-4(b, e)$ and $n=15-24(c, f)$. Raw data are available in Supplementary Table 9.

\section{Adaptive mutations predominantly improve nodulation competitiveness}

Next, we measured the effect of each adaptive mutation on nodulation competitiveness (reflecting host entry) and within-host proliferation in order to uncover which of these two symbiotic traits can explain the observed fitness gain. All mutations increasing fitness improved nodulation competitiveness of evolved clones (Fig. 3b,e and Supplementary Table 9). Gains in nodulation competitiveness ranged from 1.6 to 58 -fold, with three mutations providing the highest gains: RSc2277-V321G (58-fold), efpR-E66K (35-fold) and rhIE1-L368L (28-fold). By contrast, within-host proliferation was improved by only a subset of adaptive mutations (Fig. 3c,f and Supplementary Table 9). Only 3 mutations from lineage B (RSc1598-P455L, up ${ }^{115}$-RSc0965 and rhIE1-L368L) and 7 mutations from lineage G (efpR-E66K, RSc2277-V321G, phbB-S38F, pRALTA_0398-W719*, RSp0574-L15L, RSp0769-F416S and RSp1422-G1298E) significantly improved this fitness component. Moreover, gains in in planta proliferation were generally lower than gains in nodulation competitiveness and ranged from 1.6 to 6.4 -fold. The highest proliferation gains were produced by the five mutations RSc1598P455L (6.4-fold), efpR-E66K (3.7-fold), up ${ }^{115}$-RSc0965 (2.6-fold), RSc2277-V321G (2.5 fold) and rhIE1L368L (2.4-fold), three of which also produced the highest nodulation gains.

To investigate whether improvements in nodulation competitiveness could be due to a better colonization of the plant culture medium or rhizosphere, we measured the survival of the 28 mutants in competition with their isogenic parental strain in these two compartments. Results from these assays showed that none of the adaptive mutations improved bacterial ability to colonize the culture medium and only 4 of them, including up ${ }^{115}-\mathrm{RSc} 0965$, efpR-E66K and rhIE1-L368L, improved the colonization of the rhizosphere (Extended Data Fig. 3 and Supplementary Table 10) although by smaller factors than improvements in nodulation competitiveness. These results showed that improvements in nodulation competitiveness are generally not associated with a better colonization of the culture medium or of the rhizosphere, indicating that host entry is mainly controlled directly by the plant and 
is the dominant selective force driving the adaptation of legume symbionts in this evolution experiment.

\section{Evolutionary modelling predicts that selection for host entry drives adaptation}

The preferential selection of nodulation competitiveness in our experiment prompted us to investigate the origin of this phenomenon. In particular, we wondered if stronger selection for host entry over within-host proliferation could be a general feature of symbiotic life cycles, or, instead, if it is more likely to be a specific property of our experimental system that may arise due to genetic constraints on symbiotic traits and/or specific features of the selective regime. We used computer simulations to model the evolution of bacterial populations that cycle between two compartments: the rhizosphere, where bacteria accumulate mutations, and root nodules, where bacteria reproduce clonally (see Supplementary Information for full details on the model and Supplementary Fig. 1). In this setting (and similar to our experiment), bacterial fitness is mediated by two phenotypic components: competitiveness to enter the host (resulting in nodulation) and within-host proliferation. The nodulation step imposes a very stringent selective bottleneck during host entry, since (i) in most cases, each nodule is founded by one single bacterium ${ }^{9,27}$, (ii) there were usually between 100 and 300 nodules per lineage collected at each cycle (Supplementary Table 1) and (iii) bacterial genotypes differ in their competitive ability to form nodules (Fig. 3b,e). Once inside the host, bacteria multiply to reach a carrying capacity that is directly proportional to their proliferation fitness (no competition assumed), before returning to the rhizosphere. When following the evolution of bacterial phenotypes in populations founded by an ancestor with low initial fitness ( $10^{-4}$ fold compared to the theoretical optimum, for each phenotypic component), we observed, in most cases, a faster increase in nodulation competitiveness relative to within-host proliferation (Fig. 4a). Accordingly, the distribution of mutations that were selected at the early steps of the adaptive process was biased towards stronger improvement of nodulation over proliferation (Fig. 4b, Low fitness domain). This dominance decreased, and was sometimes reverted, as populations progressed towards high fitness values. Decreasing the strength of the nodulation bottleneck also reduced the dominance of early selection for nodulation competitiveness, until abolishing it for the largest bottleneck size tested (3000 nodules). In our experiment, the ancestral nodulating strains had a much higher potential for improvement in nodulation competitiveness relative to proliferation $\left(5 \times 10^{4}\right.$ vs. $10^{2}-10^{3}$ fold, respectively, compared to the natural symbiont $C$. taiwanensis) which could explain the faster evolution of the former trait. We performed additional simulations to test how evolutionary trajectories are affected by the genotype

292 of the ancestor. As expected, starting from a higher proliferation fitness in the ancestor further increased the overall fold effect of early selected mutations (Fig. 4c,d). In contrast, starting with a 
bioRxiv preprint doi: https//doi.org/10.1101/2022.03 03.482760; this version posted March 4, 2022. The copyright holder for this preprint (which was not certified by peer review) is the author/funder, who has granted bioRxiv a license to display the preprint in perpetuity. It is made available under aCC-BY-NC 4.0 International license.

higher initial nodulation competitiveness value of $10^{-3}$ or $10^{-2}$ in the ancestor was not sufficient to lead

a

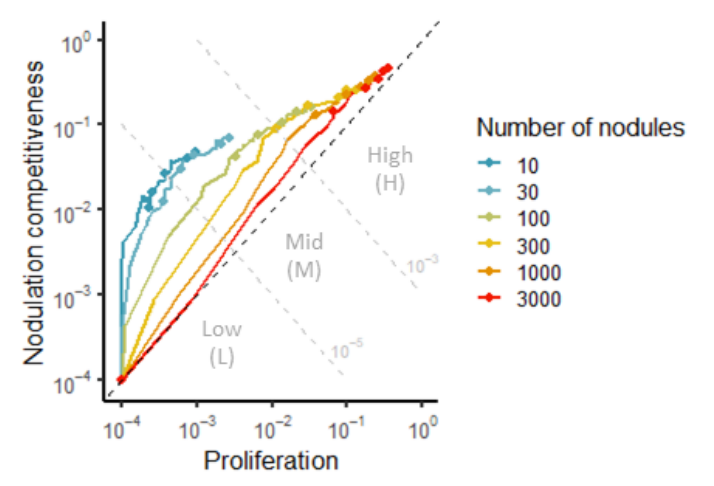

C

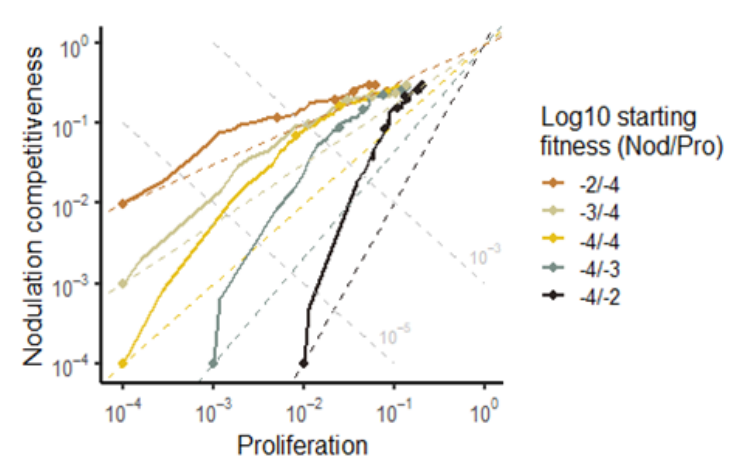

e

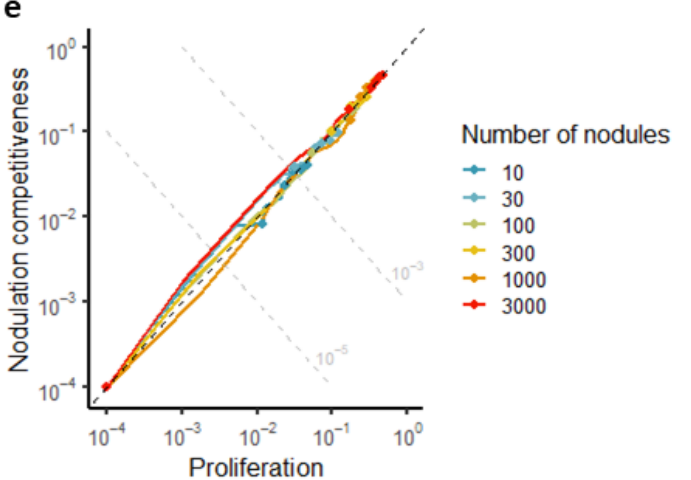

b

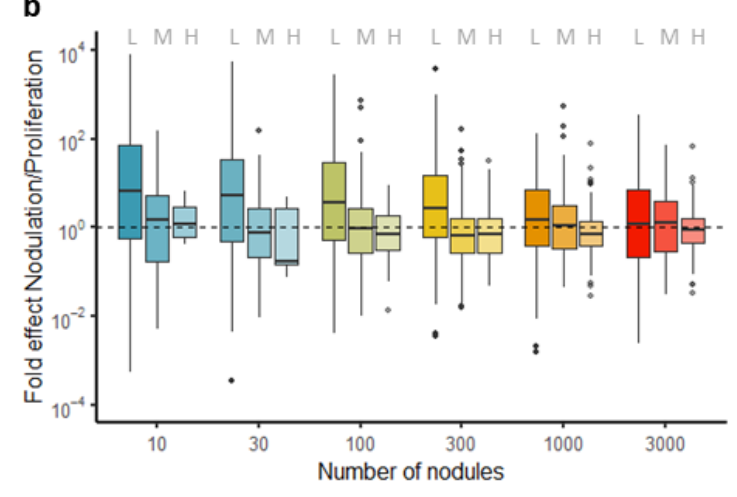

d
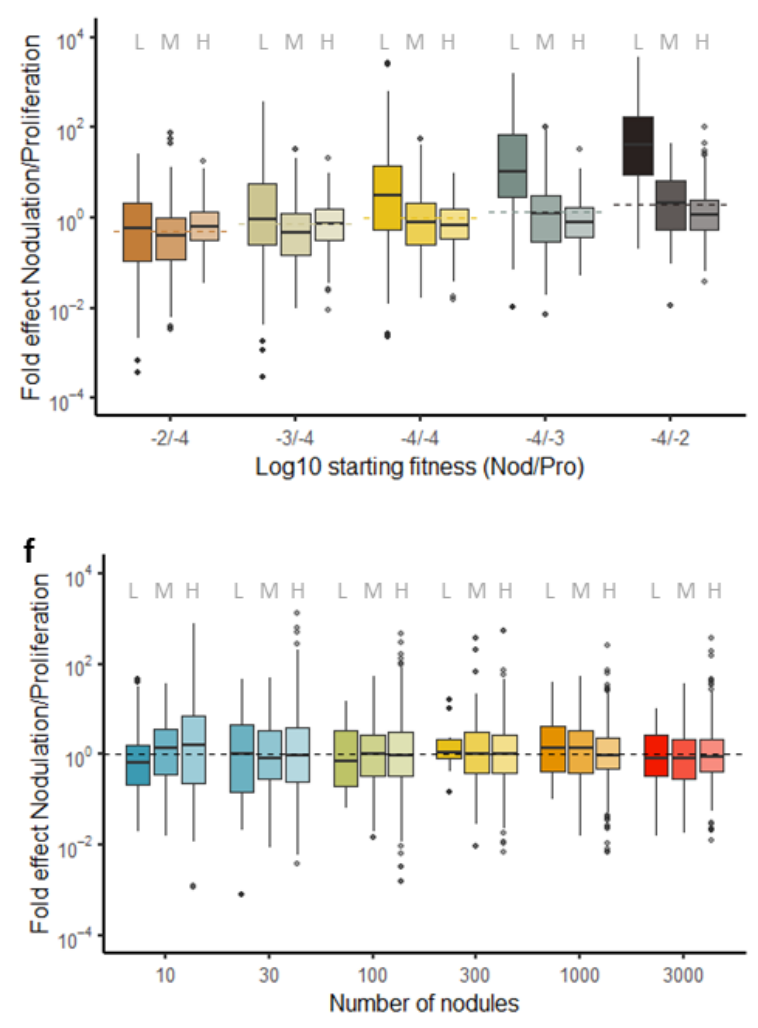

\section{Fig. 4 Relative strength of selection for nodulation competitiveness and proliferation}

a,c,e, Median fitness trajectories of 100 simulated populations evolving under different sizes of nodulation bottleneck (10 to 3000 nodules). Points indicate fitness values at cycles $0,10,20,30,40$ and 50. Dotted grey lines represent iso-fitness lines (defined as the product of nodulation competitiveness and proliferation) used to delineate 3 fitness domains: Low ( $L$; fitness $<10-5$ ), Mid (M; $10-5<$ fitness < 10-3), and High ( $\mathrm{H}$; fitness > 10-3). The black dotted line represents the diagonal, along which population would improve both phenotypic traits equally well. b,d,f, Fold effect (nodulation competitiveness effect divided by proliferation effect) of mutations that reached a frequency of at least $30 \%$ in simulated populations. Color shading indicate 
the fitness domain (Low, Mid or High) in which each group of mutations arose. Note that fold effects of mutations are

indicated even when median fitness trajectories do not reach the High fitness domain (e.g. 10 and 30 nodule lines in a) the ancestor $(c, d)$, and the size of nodulation bottleneck under a scenario where the nodulation bottleneck occurs after bacterial clonal proliferation $(e, f)$.

Finally, we wondered if the chronology of symbiotic steps impacts on selective pressures and evolutionary trajectories. We modified the simulations so that clonal multiplication of bacteria (according to their proliferation fitness) occurs before the nodulation bottleneck. Although this model is not relevant in the context of the legume-rhizobium symbiosis, it may apply to other symbiotic or pathogenic life cycles, for example when a selective bottleneck occurs during dissemination in the environment or within the host ${ }^{28,29}$ or in the case of vertically transmitted symbioses ${ }^{30}$. In this context, the dominance of nodulation competitiveness was strongly reduced, bringing the two selective forces close to equilibrium (Fig. 4e,f and Supplementary Fig. 12), in agreement with the expectation that each fitness component should have a similar influence on adaptation when they have a multiplicative effect on global fitness. Therefore, the dominance of nodulation competitiveness in the previous simulation runs is likely a consequence of the selective bottleneck occurring just after the transient hypermutagenesis phase in the rhizosphere.

Altogether, these simulations show that the selective pressures that are experienced by symbiotic bacteria are asymmetric, with a dominance of selection for nodulation competitiveness over withinhost proliferation. This asymmetry occurs when a highly diverse bacterial population from the rhizosphere is exposed to the strong, selective nodulation bottleneck, allowing the efficient selection of the most competitive clones by the host plant. In our evolution experiment, the fact that the ancestor has a lower nodulation competitiveness value compared to proliferation (relative to that of the wild-type rhizobium $C$. taiwanensis) is an additional factor expected to have contributed to the stronger selection on nodulation competitiveness.

\section{Discussion}

Horizontally transmitted symbiotic bacteria have complex lifecycles during which they have to face multiple environmental constraints, both outside and inside the host. Assessing the relative influence of each of these selective pressures on the adaptive trajectories of emerging symbiotic bacteria is critically needed to better understand the eco-evolutionary dynamics of microbial populations ${ }^{5}$. Here we show that selection on competitiveness for host entry is the dominant selective pressure shaping early adaptation of new rhizobia. This trait, mainly mediated by the plant, improved very fast during our evolution experiment, to a level comparable to that of the Mimosa rhizobium 

competitiveness. Evolutionary simulations led us to propose that the dominance of the selection on host entry over the selection on within-host proliferation that we observed in our system is strongly dependent on the strength of the selective nodulation bottleneck. These results shed light on the importance of selective bottlenecks in shaping the evolutionary trajectories of emerging microbial symbionts. Most theoretical ${ }^{31,32}$ and experimental ${ }^{33-38}$ works on the influence of bottlenecks on microbial adaptation have focused on non-selective bottlenecks that randomly purge genetic diversity and reduce the efficiency of natural selection. Yet, another aspect of bottlenecks emerges when considering that transmission and host colonization can be, at least partially, dependent on microbial genotype, prompting us to consider infection bottlenecks as selective events ${ }^{39}$. Selective bottlenecks have already been described during virus infections ${ }^{28,40-43}$ but received less attention in bacteria, except for their role in the evolution of phenotypic heterogeneity ${ }^{17,44-46}$. However selective bottlenecks are likely common both in mutualistic and pathogenic interactions. Indeed, potentially all symbiotic microorganisms (whether commensal, pathogenic or mutualists) experience bottlenecks of various intensities (but sometimes as strong as only a few cells ${ }^{47}$ ) during their life cycles ${ }^{48,49}$. Given that microbes rely on complex genetic networks to disseminate and infect their hosts ${ }^{7,50-53}$, one can expect these traits to display extensive genetic and phenotypic variation within populations and evolve through competition with other members of the microbiome. Interestingly, strong selection for host entry in experimentally evolved symbionts was recently observed in several studies ${ }^{54-56}$, reinforcing the idea that selective bottlenecks for host entry may play a crucial role in the evolution of diverse symbiotic interactions.

The molecular dissection of bacterial adaptation confirmed that the genetic architecture of rhizobial symbiotic traits is complex, as it involves many different genes and physiological functions ${ }^{57,58}$. Our study also unveiled a new characteristic of the symbiotic genotype-to-phenotype map: the recurrent genetic coupling between nodulation competitiveness and within-host proliferation. Indeed, all the 10 identified mutations that improved within-host proliferation also improved nodulation competiveness, while the reverse was not true. This result corroborates our previous data showing that mutations promoting nodule cell infection always improved nodule formation ${ }^{14,59}$. It is conceivable that the intracellular release and proliferation of rhizobia in nodule cells is dependent on the efficiency of the earliest symbiotic events, i. e. the entry and progression of bacteria in infection threads and the concomitant divisions of nodule cells preparing for the accommodation of bacteria. Consistently, delayed progression of infection threads across the root cell layers was shown to impair the release of bacteria in nodule cells ${ }^{60}$. Moreover plant receptors (LYK3, NFR1, Sym37, SYMRK) and transcription 376 factors (NIN, ERN1, ERN2) involved in both nodule organogenesis and rhizobial intracellular infection 
the plant at the two entry points, the root hairs (host entry) and the nodule cells (within-host proliferation). Yet, this coupling is not strict, since some mutations increase nodulation competitiveness without increasing proliferation. These mutations may target bacterial functions specifically required for nodulation. Alternatively, the fact that most mutations with an effect on proliferation are the ones with the highest contribution to nodulation competitiveness could be explained by a threshold effect, i.e. the existence of a minimal level above which mutations that increase nodulation competitiveness also increase proliferation. Surprisingly, mutations only improving proliferation were not found in our study. At this stage, we cannot distinguish between two hypotheses, namely if these mutations do not exist (proliferation is always linked to nodulation and host entry) or if they were not yet selected in this experiment due to the dominance of nodulation competitiveness in the first steps of adaptation. Future functional analyses of the adaptive mutations identified in this study will expand our understanding of the molecular bases of nodulation competitiveness in rhizobia ${ }^{65-67}$, and its relationship to within-host proliferation. Interestingly, these two traits primarily selected by the plant, although necessary for the symbiotic interaction, are not, in themselves, beneficial for the host. Including nitrogen fixation and host growth promotion in the analysis of genetic couplings between the different symbiotic traits, will be fundamental to better understand the evolution of mutualism ${ }^{67}$.

In all lineages of this experiment, the rate of adaptation was very high during the first cycles of evolution and then tended to decrease over time. This classical pattern of evolution, due to diminishing return epistasis among beneficial mutations ${ }^{24,68,69}$, was likely favored by the low initial fitness values of nodulating ancestors compared to the natural symbiont $C$. taiwanensis allowing the rapid acquisition of highly beneficial mutations during the first cycles (Table 1). Elevated mutation rate in the rhizosphere, prior to the entry of bacteria in roots ${ }^{18}$, has also played an important role in the dynamic of adaptation by fueling the extensive genetic diversification of bacteria and exposing these populations to plant-mediated selection. A probable consequence of high mutation rate combined to strong selection is the presence of large cohorts of mutations that carry multiple beneficial mutations along with some neutral or slightly deleterious ones. Mutational cohorts were already described in previous evolution experiments ${ }^{21,70}$, with cases of cohorts carrying 2 co-driver mutations ${ }^{22}$. Here we identified up to 7 adaptive mutations in one of our cohorts. The occurrence of multi-driver mutations per cohort might be explained by the nested, sequential fixation of multiple adaptive mutations in one lineage before it reaches detectable frequency ( $5 \%$ in our case) ${ }^{22}$. This phenomenon, creating a 'travelling wave' of adaptation ${ }^{71}$, was recently observed with high-resolution sequencing ${ }^{23}$ and is possibly amplified by the strong selection/high mutation regime of our experiment. Alternatively, it was proposed that, in the case of hypermutagenesis, the likelihood of multiple adaptive mutations 
arising simultaneously in a given genome becomes non-negligible and might promote saltational evolution ${ }^{72}$. In conclusion, our work shows that the relative strengths of the selective forces imposed by hosts strongly influence the evolutionary trajectory of microbial populations. Selection for host entry is likely the predominant life-history trait selected during the evolution of new horizontally-transmitted symbiotic interactions, such as the rhizobium-legume symbiosis. This effect emerges from the presence of a selective bottleneck at host entry, although its predominance can be modulated by other genetic and ecological factors. Investigating the role of selective bottlenecks in other symbiotic interactions will improve our ability to manage and predict the eco-evolutionary dynamics of hostassociated microbial populations.

\section{Methods}

\section{Bacterial strains and growth conditions}

Strains and plasmids used in this study are listed in Supplementary Table 11. Cupriavidus taiwanensis strains were grown at $28^{\circ} \mathrm{C}$ on tryptone-yeast (TY) medium ${ }^{73}$. Ralstonia solanacearum strains were grown at $28^{\circ} \mathrm{C}$ either on $\mathrm{BG}$ medium ${ }^{74}$ or on minimal MP medium ${ }^{75}$ supplemented with $2 \%$ glycerol. Escherichia coli strains were grown at $37^{\circ} \mathrm{C}$ on Luria-Bertani medium ${ }^{76}$. Antibiotics were used at the following concentrations: trimethoprim at $100 \mathrm{gg} \mathrm{ml}^{-1}$, spectinomycin at $40 \mathrm{\mu g} \mathrm{ml}^{-1}$, streptomycin at $200 \mu \mathrm{g} \mathrm{ml}^{-1}$ and kanamycin at $25 \mathrm{\mu g} \mathrm{ml}^{-1}$ (for E. coli) or $50 \mu \mathrm{g} \mathrm{ml}^{-1}$ (for R. solanacearum).

\section{Experimental evolution}

Symbiotically evolved clones and populations were generated as previously described ${ }^{16}$. Five lineages, two ( $\mathrm{B}$ and $\mathrm{F}$ ) derived from the CBM212 ancestor, two ( $\mathrm{G}$ and $\mathrm{K}$ ) derived from the CBM349 ancestor and one (M) derived from the CBM356 ancestor, previously evolved for 16 cycles were further evolved until cycle 35. For each lineage, at each cycle, 30 plants were inoculated with nodule bacterial populations from the previous cycle. Twenty-one days after inoculation, all nodules from the 30 plants were pooled, surface-sterilized with $2.6 \%$ sodium hypochlorite for $15 \mathrm{~min}$, rinsed with sterile water and crushed. Then, $10 \%$ of the nodule crush was used to inoculate a new set of 30 plants the same day. Serial dilutions of each nodule crush were plated and one clone was randomly selected from the highest dilution and purified. The selected clones, the rest of the nodule crushes, and a 24-h culture of bacteria from an aliquot of nodule crushes were stored at $-80^{\circ} \mathrm{C}$ at each cycle.

\section{Sequencing bacterial populations and clones}


Aliquots of frozen nodule bacterial populations or single colonies from purified clones were grown overnight in BG medium supplemented with trimethoprim. Bacterial DNA was extracted from $1 \mathrm{ml}$ of culture using the Wizard genomic DNA purification kit (Promega). Evolved population DNAs were sequenced at the GeT-PlaGe core facility (https://get.genotoul.fr/), INRAE Toulouse. DNA-seq libraries have been prepared according to Illumina's protocols using the Illumina TruSeq Nano DNA HT Library Prep Kit. Briefly, DNA was fragmented by sonication, size selection was performed using SPB beads (kit beads) and adaptators were ligated to be sequenced. Library quality was assessed using an Advanced Analytical Fragment Analyzer and libraries were quantified by GPCR using the Kapa Library Quantification Kit (Roche). Sequencing has been performed on a NovaSeq6000 S4 lane (Illumina, California, USA) using a paired-end read length of 2×150 pb with the Illumina NovaSeq Reagent Kits. Evolved clones were re-sequenced either by C.E.A/IG/Genoscope using the Illumina GA2X technology (clones B8, B16, F16, G8, G16, K16 and M16), or by the GeT_PlaGe core facility using the Illumina technology HiSeq2000 (clones B1, B2, B3, B4, B5, B6, B7, B9, B10, B11, B12, B13, B14, B15, G1, G2, G3, G4, G5, G6, G7, G9, G10, G11, G12, G13, G14, and G15), or HiSeq3000 (clones B20, B25, B30, G25 and G30), or NovaSeq6000 (clones B35, F35, G35, K35 and M35).

\section{Detection of mutations and molecular analyses}

Sequencing reads from NovaSeq6000 runs (whole-populations and clones from cycle 35) were mapped on the chimeric reference genome of the ancestral strain, comprising $R$. solanacearum GMI1000 chromosome (GenBank accession number: NC_003295.1) and megaplasmid (NC_003296.1) together with C. taiwanensis symbiotic plasmid pRalta (CU633751). Mutations were detected using breseq v0.33.1(Ref. ${ }^{77}$ ) with default parameters, either using the polymorphism mode (for whole-population sequences) or the consensus mode (for individual clones). Mutation lists were curated manually in order to remove mutations present in the ancestral strains as well as false positive hits arising from reads misalignments in low complexity regions. Moreover, alleles showing aberrant trajectories in the time-course whole-population sequencing data were checked manually, by inspecting either breseq output files and/or read alignments with IGV78, and corrected as needed. In this work, we focused our attention on SNPs and indels detected above $5 \%$ frequency in the populations. Recombinations, rearrangements and IS movements were not analyzed exhaustively. Mutations detected in populations are listed in Supplementary Tables 4-8.

Sequencing data of the remaining clones were analyzed as described previously ${ }^{26}$ with the PALOMA bioinformatics pipeline implemented in the Microscope platform ${ }^{79}$. The complete list of mutational events generated for these clones are available on the Microscope platform (https://mage.genoscope.cns.fr/microscope/expdata/NGSProjectEvo.php, SYMPA tag). Mutations detected in clones are listed in Supplementary Tables 12-16. 
481

482

483

484

485

486

487

488

489

490

491

492

493

494

495

496

497

498

499

500

501

502

503

504

505

506

507

508

509

510

511

512

513

514

515

In order to identify mutations forming temporal clusters (cohorts) through populations, we selected mutations having a frequency of $30 \%$ in at least one cycle and clustered their frequency using the hclust function in R v3.6.1 ${ }^{80}$. Then, we separated cohorts using a cutoff distance of 0.3.

Sub-population genealogies in lineages B and G, shown as Muller plots in Extended Data Figure 2, were reconstructed by comparing mutations found in cohorts and in individually sequenced clones. Cohorts for which ancestry cannot be ascertained (mutations not found in any clone) were not included in these plots. Relative frequencies of genotypes were calculated manually and plotted with ggMuller ${ }^{81}$. $\mathrm{G}$ scores were calculated as described ${ }^{20}$. Since we found that synonymous mutations can be adaptive, we used all types of mutations in coding regions to calculate this statistics. Moreover, we included all mutations beyond $5 \%$ frequency in this analysis since we assumed that strong clonal interference may prevent adaptive mutations to rise to high frequency. To evaluate statistical significance of $\mathrm{G}$ scores, we ran 1,000 simulations where the total number of mutations used for the calculation of the observed G scores (3330) were randomly attributed in the coding genes according to their respective length to compute simulated $\mathrm{G}$ scores for each bacterial gene. The sum of simulated $\mathrm{G}$ scores was compared to the observed sum and we computed a $Z$ score and $P$-value from these simulated $G$ statistics. These simulations were also used to compute mean $\mathrm{G}$ scores for each gene, and to calculate the associated $Z$ scores and $P$-values (adjusted using a Bonferroni correction).

\section{Reconstruction of mutations in evolved clones}

Mutant alleles and constitutively expressed reporter genes (GFPuv, mCherry) were introduced into Ralstonia evolved clones by co-transformation using the MuGent technique ${ }^{26,82}$. Briefly, two DNA fragments, the first one carrying an antibiotic (kanamycin) resistance gene prepared from the pRCKPps-GFP or pRCK-Pps-mCherry linearized plasmids allowing the integration of the resistance gene in the intergenic region downstream the gImS gene, and the second one carrying the mutation to be introduced prepared by PCR amplification of a $6 \mathrm{~kb}$ region using genomic DNA of evolved clones as template and high fidelity Phusion polymerase (ThermoFisher Scientific), were co-transformed into naturally competents cells of Ralstonia evolved clones. Co-transformants resistant to kanamycin were screened by PCR using primers specifically amplifying mutant or wild-type alleles and verified by Sanger sequencing. Primers used in mutation reconstructions are listed in Supplementary Table 17.

\section{Analyses of symbiotic phenotypes on $M$. pudica}

Mimosa pudica seeds from LIPME production (2019 batch) were sterilized as described ${ }^{83}$ by immersion in $95 \% \mathrm{H}_{2} \mathrm{SO}_{4}$ during 15 minutes and $2.4 \%$ sodium hypochlorite solution during 10 minutes and rinsed in sterile distilled water 5 times. Seeds were soaked in sterile water at $28^{\circ} \mathrm{C}$ under agitation for 24 hours and then deposited on soft agar $(9.375 \mathrm{~g} / \mathrm{L})$ and incubated at $28^{\circ} \mathrm{C}$ during 24 more hours in 
darkness. Then, seedlings were cultivated in glass tubes (2 seedlings/tube) under $\mathrm{N}$-free conditions, each tube containing $20 \mathrm{~mL}$ of solid Fahraeus medium ${ }^{84}$ and $40 \mathrm{~mL}$ of liquid Jensen medium ${ }^{85}$ diluted 1:4 with sterile water. Plants were incubated in a culture chamber at $28^{\circ} \mathrm{C}$ under a photoperiod day/night of $16 \mathrm{~h} / 8 \mathrm{~h}$.

For in planta relative fitness and nodulation competitiveness assays, two strains of Ralstonia solanacearum expressing differential constitutive fluorophores (GFPuv or mCherry) or one strain of $R$. solanacearum and one strain of $C$. taiwanensis, both expressing differential antibiotic resistance (streptomycin and trimethoprim), were co-inoculated onto M. pudica plantlets grown for 3 to 4 days in the culture chamber. Both strains were inoculated in equivalent proportion ( $c a .5 \times 10^{5}$ bacteria of each strain per plant) except for the comparisons of the Ralstonia nodulating ancestors with $C$. taiwanensis where strains were mixed in a 1000:1 ratio (ca. $5 \times 10^{3}$ bacteria of $C$. taiwanensis and $c a$. $5 \times 10^{5}$ bacteria of nodulating ancestors per plant). Nodules were harvested 21 days after inoculation, surface sterilized by immersion in $2.4 \%$ sodium hypochlorite solution for 15 minutes and rinsed with sterile water. For in planta relative fitness measurements, sterilized nodules from 20 plants (see the exact number of harvested nodules in Supplementary Table 1) were pooled and crushed in $1 \mathrm{~mL}$ of sterile water, diluted and spread on selective solid medium using an easySpiral automatic plater (Interscience). After 2-day incubation at $28^{\circ} \mathrm{C}$, colonies were screened either by plating on selective medium in case of co-inoculations of Ralstonia evolved clones with C. taiwanensis or based on fluorescence using a stereo zoom microscope (AxioZoom V16, Zeiss, Oberkochen, Germany) for coinoculations of Ralstonia evolved clones with Ralstonia reconstructed mutants. For nodulation competitiveness assays, ca. 96 individual nodules per experiment were crushed separately in 96-well microtiter plates and droplets were deposited on selective medium. Nodule occupancy was determined by screening bacteria grown in the droplets either on selective medium or based on their fluorescence as described for fitness measurements. Both nodulation competitiveness and relative fitness assays were measured in at least three independent experiments. Competitive indexes $(\mathrm{Cl})$ were calculated by dividing the ratio of the number of test strain (evolved strains or reconstructed mutants) vs. reference strain (C. taiwanensis or evolved parental strains, respectively) in nodules normalized by the inoculum ratio. $\mathrm{Cl}$ values above 1 were transformed by their inverse and compared to the value 1 using a one-sided Student $t$-test $(P<0.05)$.

For within-host proliferation assays, plantlets were inoculated with a single strain $\left(5 \times 10^{5}\right.$ bacteria per plant). In each experiment, nodules from 6 individual plants were collected separately 21 days after inoculation, surface sterilized for 15 minutes in a $2.4 \%$ sodium hypochlorite solution, rinsed with sterile water and crushed in $1 \mathrm{ml}$ of sterile water. Dilutions of nodule crushes were plated on selective solid medium using an easySpiral automatic plater (Interscience). Two days after incubation at $28^{\circ} \mathrm{C}$, the number of colonies was counted. Within-host proliferation was estimated as the number of bacteria 
551

552

553

554

555

556

557

558

559

560

561

562

563

564

565

566

567

568

569

570

571

572

573

574

575

576

577

578

579

580

581

582

583

584

per nodule. For each strain, 15 to 24 measurements from three independent experiments were performed. Pairwise comparisons of proliferation values were compared using a two-sided Wilcoxon rank sum test.

For Jensen culture medium and rhizosphere colonization assays, plantlets were co-inoculated with pair of strains expressing differential constitutive fluorophores (GFPuv or mCherry) in equivalent proportion $\left(5 \times 10^{6}\right.$ bacteria of each strain per plant). Seven days after inoculation, bacteria present in the culture medium were diluted and plated on selective solid medium using an easySpiral automatic plater (Interscience). Bacteria attached to the roots were resuspended in $4 \mathrm{ml}$ of sterile water by strongly vortexing for 1 minute, diluting and plating on selective medium using an easySpiral automatic plater (Interscience). Two days after incubation at $28^{\circ} \mathrm{C}$, colonies were screened for fluorescence using a stereo zoom microscope (AxioZoom V16, Zeiss, Oberkochen, Germany). Competitive indexes (CI) for Jensen medium or rhizosphere colonization were calculated as described above. $\mathrm{Cl}$ values were compared to the value 1 using a two-sided Wilcoxon rank sum test with Benjamini-Hochberg correction.

\section{Modelling/simulations}

Full details on evolutionary simulations are available as Supplementary Information.

\section{Data availability}

Sequencing data are available under NCBI SRA BioProject ID PRJNA788708 and SRP353965. Raw experimental data are available as Supplementary Information. Raw data generated from computer simulations are deposited on the Data INRAE dataverse (https://doi.org/10.15454/QYB2S9).

\section{Code availability}

Computer code used to analyze genomic data and to perform computer simulations are deposited on the Data INRAE dataverse (https://doi.org/10.15454/QYB2S9).

\section{References}

1. Drew, G. C., Stevens, E. J. \& King, K. C. Microbial evolution and transitions along the parasitemutualist continuum. Nat. Rev. Microbiol. 19, 623-638, doi:10.1038/s41579-021-00550-7 (2021).

2. Nandasena, K. G., O'Hara, G. W., Tiwari, R. P., Sezmis, E. \& Howieson, J. G. In situ lateral transfer of symbiosis islands results in rapid evolution of diverse competitive strains of mesorhizobia suboptimal in symbiotic nitrogen fixation on the pasture legume Biserrula pelecinus L. Environ. Microbiol. 9, 2496-2511, doi:10.1111/j.1462-2920.2007.01368.x (2007). 
585

586

587

588

589

590

591

592

593

594

595

596

597

598

599

600

601

602

603

604

605

606

607

608

609

610

611

612

613

614

615

616

617

3. Longdon, B. et al. The causes and consequences of changes in virulence following pathogen host shifts. PLoS Pathog. 11, e1004728, doi:10.1371/journal.ppat.1004728 (2015).

4. Bonneaud, C. \& Longdon, B. Emerging pathogen evolution: using evolutionary theory to understand the fate of novel infectious pathogens. EMBO Rep. 21, e51374, doi:10.15252/embr.202051374 (2020).

5. Obeng, N., Bansept, F., Sieber, M., Traulsen, A. \& Schulenburg, H. Evolution of microbiota-host associations: the microbe's perspective. Trends Microbiol. 29, 779-787, doi:10.1016/j.tim.2021.02.005 (2021).

6. Robinson, C. D., Bohannan, B. J. \& Britton, R. A. Scales of persistence: transmission and the microbiome. Curr. Opin. Microbiol. 50, 42-49, doi:10.1016/j.mib.2019.09.009 (2019).

7. Wheatley, R. M. et al. Lifestyle adaptations of Rhizobium from rhizosphere to symbiosis. Proc. Natl. Acad. Sci. U. S. A. 117, 23823-23834, doi:10.1073/pnas.2009094117 (2020).

8. Gage, D. J. Analysis of infection thread development using Gfp- and DsRed-expressing Sinorhizobium meliloti. J. Bacteriol. 184, 7042-7046, doi:10.1128/jb.184.24.7042-7046.2002 (2002).

9. Gage, D. J. Infection and invasion of roots by symbiotic, nitrogen-fixing rhizobia during nodulation of temperate legumes. Microbiol. Mol. Biol. Rev. 68, 280-300, doi:10.1128/mmbr.68.2.280300.2004 (2004).

10. Coba de la Peña, T., Fedorova, E., Pueyo, J. J. \& Lucas, M. M. The symbiosome: legume and rhizobia co-evolution toward a nitrogen-fixing organelle? Front. Plant Sci. 8, 2229, doi:10.3389/fpls.2017.02229 (2017).

11. Hirsch, A. M. et al. Rhizobium meliloti nodulation genes allow Agrobacterium tumefaciens and Escherichia coli to form pseudonodules on alfalfa. J. Bacteriol. 158, 1133-1143 (1984).

12. Abe, M. et al. Transfer of the symbiotic plasmid from Rhizobium leguminosarum biovar trifolii to Agrobacterium tumefaciens. J. Gen. Appl. Microbiol. 44, 65-74 (1998).

13. Nakatsukasa, H. et al. Transposon mediation allows a symbiotic plasmid of Rhizobium leguminosarum bv. trifolii to become a symbiosis island in Agrobacterium and Rhizobium. J. Gen. Appl. Microbiol. 54, 107-118, doi:10.2323/jgam.54.107 (2008).

14. Marchetti, M. et al. Experimental evolution of a plant pathogen into a legume symbiont. PLoS Biol. 8, doi:e1000280 10.1371/journal.pbio.1000280 (2010).

15. Masson-Boivin, C., Giraud, E., Perret, X. \& Batut, J. Establishing nitrogen-fixing symbiosis with legumes: how many rhizobium recipes? Trends Microbiol. 17, 458-466, doi:10.1016/j.tim.2009.07.004 (2009). 
16. Marchetti, M. et al. Experimental evolution of rhizobia may lead to either extra- or intracellular symbiotic adaptation depending on the selection regime. Mol. Ecol. 26, 1818-1831, doi:10.1111/mec.13895 (2017).

17. Moxon, R. \& Kussell, E. The impact of bottlenecks on microbial survival, adaptation, and phenotypic switching in host-pathogen interactions. Evolution 71, 2803-2816, doi:10.1111/evo.13370 (2017).

18. Remigi, P. et al. Transient hypermutagenesis accelerates the evolution of legume endosymbionts following horizontal gene transfer. PLoS Biol. 12, e1001942, doi:10.1371/journal.pbio.1001942 (2014).

19. Lynch, M. et al. Genetic drift, selection and the evolution of the mutation rate. Nat. Rev. Genet. 17, 704-714, doi:10.1038/nrg.2016.104 (2016).

20. Tenaillon, O. et al. Tempo and mode of genome evolution in a 50,000-generation experiment. Nature 536, 165-170, doi:10.1038/nature18959 (2016).

21. Lang, G. I. et al. Pervasive genetic hitchhiking and clonal interference in forty evolving yeast populations. Nature 500, 571-574, doi:10.1038/nature12344 (2013).

22. Buskirk, S. W., Peace, R. E. \& Lang, G. I. Hitchhiking and epistasis give rise to cohort dynamics in adapting populations. Proc. Natl. Acad. Sci. U. S. A. 114, 8330-8335, doi:10.1073/pnas.1702314114 (2017).

23. Nguyen $\mathrm{Ba}, \mathrm{A}$. N. et al. High-resolution lineage tracking reveals travelling wave of adaptation in laboratory yeast. Nature 575, 494-499, doi:10.1038/s41586-019-1749-3 (2019).

24. McDonald, M. J. Microbial experimental evolution - a proving ground for evolutionary theory and a tool for discovery. EMBO Rep. 20, e46992, doi:10.15252/embr.201846992 (2019).

25. Bailey, S. F., Alonso Morales, L. A. \& Kassen, R. Effects of synonymous mutations beyond codon bias: the evidence for adaptive synonymous substitutions from microbial evolution experiments. Genome Biol. Evol. 13, doi:10.1093/gbe/evab141 (2021).

26. Capela, D. et al. Recruitment of a lineage-specific virulence regulatory pathway promotes intracellular infection by a plant pathogen experimentally evolved into a legume symbiont. Mol. Biol. Evol. 34, 2503-2521, doi:10.1093/molbev/msx165 (2017).

27. Daubech, B. et al. Spatio-temporal control of mutualism in legumes helps spread symbiotic nitrogen fixation. eLife 6, doi:10.7554/eLife.28683 (2017).

28. Joseph, S. B., Swanstrom, R., Kashuba, A. D. \& Cohen, M. S. Bottlenecks in HIV-1 transmission: insights from the study of founder viruses. Nat. Rev. Microbiol. 13, 414-425, doi:10.1038/nrmicro3471 (2015). 
29. Hullahalli, K. \& Waldor, M. K. Pathogen clonal expansion underlies multiorgan dissemination and organ-specific outcomes during murine systemic infection. elife 10, doi:10.7554/eLife.70910 (2021).

30. Perreau, J., Zhang, B., Maeda, G. P., Kirkpatrick, M. \& Moran, N. A. Strong within-host selection in a maternally inherited obligate symbiont: Buchnera and aphids. Proc. Natl. Acad. Sci. U. S. A. 118, doi:10.1073/pnas.2102467118 (2021).

31. Bergstrom, C. T., McElhany, P. \& Real, L. A. Transmission bottlenecks as determinants of virulence in rapidly evolving pathogens. Proc. Natl. Acad. Sci. U. S. A. 96, 5095-5100, doi:10.1073/pnas.96.9.5095 (1999).

32. LeClair, J. \& Wahl, L. The impact of population bottlenecks on microbial adaptation. J. Stat. Physics 172, 114-125, doi:10.1007/s10955-017-1924-6 (2018).

33. Izutsu, M., Lake, D., Matson, Z., Dodson, J. \& Lenski, R. Effects of periodic bottlenecks on the dynamics of adaptive evolution in microbial populations. bioRxiv doi:10.1101/2021.12.29.474457 (2022).

34. Wein, T. \& Dagan, T. The Effect of population bottleneck size and selective regime on genetic diversity and evolvability in bacteria. Genome Biol. Evol. 11, 3283-3290, doi:10.1093/gbe/evz243 (2019).

35. Garoff, L. et al. Population bottlenecks strongly influence the evolutionary trajectory to fluoroquinolone resistance in Escherichia coli. Mol. Biol. Evol. 37, 1637-1646, doi:10.1093/molbev/msaa032 (2020).

36. Mahrt, N. et al. Bottleneck size and selection level reproducibly impact evolution of antibiotic resistance. Nat. Ecol. Evol. 5, 1233-1242, doi:10.1038/s41559-021-01511-2 (2021).

37. Windels, E. M. et al. Population bottlenecks strongly affect the evolutionary dynamics of antibiotic persistence. Mol. Biol. Evol. 38, 3345-3357, doi:10.1093/molbev/msab107 (2021).

38. Sousa, A. et al. Recurrent Reverse Evolution Maintains Polymorphism after Strong Bottlenecks in Commensal Gut Bacteria. Mol. Biol. Evol. 34, 2879-2892, doi:10.1093/molbev/msx221 (2017).

39. Handel, A. \& Bennett, M. R. Surviving the bottleneck: transmission mutants and the evolution of microbial populations. Genetics 180, 2193-2200, doi:10.1534/genetics.108.093013 (2008).

40. Wilker, P. R. et al. Selection on haemagglutinin imposes a bottleneck during mammalian transmission of reassortant H5N1 Influenza viruses. Nat. Commun. 4, 2636, doi:10.1038/ncomms3636 (2013).

41. Moncla, L. H. et al. Selective bottlenecks shape evolutionary pathways taken during mammalian adaptation of a 1918-like avian Influenza virus. Cell Host Microbe 19, 169-180, doi:10.1016/j.chom.2016.01.011 (2016). 
685

686

687

688

689

690

691

692

693

694

695

696

697

698

699

700

701

702

703

704

705

706

707

708

709

710

711

42. Sobel Leonard, A. et al. Deep sequencing of Influenza a virus from a human challenge study reveals a selective bottleneck and only limited intrahost genetic diversification. J. Virol. 90, 11247-11258, doi:10.1128/JVI.01657-16 (2016).

43. Huang, S. W. et al. A selective bottleneck shapes the evolutionary mutant spectra of Enterovirus A71 during viral dissemination in humans. J. Virol. 91, doi:10.1128/JVI.01062-17 (2017).

44. Libby, E. \& Rainey, P. B. Exclusion rules, bottlenecks and the evolution of stochastic phenotype switching. Proc. Biol. Sci. 278, 3574-3583, doi:10.1098/rspb.2011.0146 (2011).

45. De Ste Croix, M. et al. Selective and non-selective bottlenecks as drivers of the evolution of hypermutable bacterial loci. Mol. Microbiol. 113, 672-681, doi:10.1111/mmi.14453 (2020).

46. Schmutzer, M. \& Wagner, A. Gene expression noise can promote the fixation of beneficial mutations in fluctuating environments. PLoS Comput. Biol. 16, e1007727, doi:10.1371/journal.pcbi.1007727 (2020).

47. Gama, J. A., Abby, S. S., Vieira-Silva, S., Dionisio, F. \& Rocha, E. P. Immune subversion and quorumsensing shape the variation in infectious dose among bacterial pathogens. PLoS Pathog. 8, e1002503, doi:10.1371/journal.ppat.1002503 (2012).

48. Abel, S., zur Wiesch, PA, Davis, B. \& Waldor, M. Analysis of bottlenecks in experimental models of infection. PLoS Pathogen 11(6):e1004823doi:10.1371/journal.ppat.1004823 (2015).

49. Papkou \& A Gokhale, C. T., A Schulenburg, H. Host-parasite coevolution: why changing population size matters. Zoology 117, 330-338, doi:10.1016/j.zool.2016.02.001 (2016).

50. Cossart, P. \& Sansonetti, P. J. Bacterial invasion: the paradigms of enteroinvasive pathogens. Science 304, 242-248, doi:10.1126/science.1090124 (2004).

51 Visick, K. L., Stabb, E. V. \& Ruby, E. G. A lasting symbiosis: how Vibrio fischeri finds a squid partner and persists within its natural host. Nat. Rev. Microbiol. 19, 654-665, doi:10.1038/s41579-02100557-0 (2021).

52. Rowe, H. M. et al. Bacterial factors required for transmission of Streptococcus pneumoniae in mammalian hosts. Cell Host Microbe 25, 884-891.e886, doi:10.1016/j.chom.2019.04.012 (2019).

53. Bright, M. \& Bulgheresi, S. A complex journey: transmission of microbial symbionts. Nat. Rev. Microbiol. 8, 218-230, doi:10.1038/nrmicro2262 (2010).

54. Robinson, C. D. et al. Experimental bacterial adaptation to the zebrafish gut reveals a primary role for immigration. PLoS Biol. 16, e2006893, doi:10.1371/journal.pbio.2006893 (2018).

55. Bacigalupe, R., Tormo-Mas, M., Penadés, J. R. \& Fitzgerald, J. R. A multihost bacterial pathogen overcomes continuous population bottlenecks to adapt to new host species. Sci. Adv. 5, eaax0063, doi:10.1126/sciadv.aax0063 (2019). 
56. Batstone, R. T., O'Brien, A. M., Harrison, T. L. \& Frederickson, M. E. Experimental evolution makes microbes more cooperative with their local host genotype. Science 370, 476-478, doi:10.1126/science.abb7222 (2020).

57. Pobigaylo, N., Szymczak, S., Nattkemper, T. W. \& Becker, A. Identification of genes relevant to symbiosis and competitiveness in Sinorhizobium meliloti using signature-tagged mutants. Mol. Plant Microbe Interact. 21, 219-231, doi:10.1094/MPMI-21-2-0219 (2008).

58. Flores-Tinoco, C. E., Christen, M. \& Christen, B. Co-catabolism of arginine and succinate drives symbiotic nitrogen fixation. Mol. Syst. Biol. 2020 16, e9419. doi: 10.15252/msb.20199419. (2019)

59. Guan, S. H. et al. Experimental evolution of nodule intracellular infection in legume symbionts. ISME J. 7, 1367-1377, doi:10.1038/ismej.2013.24 (2013).

60. Xiao, T. T. et al. Fate map of Medicago truncatula root nodules. Development 141, 3517-3528, doi:10.1242/dev.110775 (2014).

61. Moling, S. et al. Nod factor receptors form heteromeric complexes and are essential for intracellular infection in medicago nodules. Plant Cell 26, 4188-4199, doi:10.1105/tpc.114.129502 (2014).

62. Cerri, M. R. et al. The symbiosis-related ERN transcription factors act in concert to coordinate rhizobial host root infection. Plant Physiol. 171, 1037-1054, doi:10.1104/pp.16.00230 (2016).

63. Liu, J. et al. NIN is essential for development of symbiosomes, suppression of defence and premature senescence in Medicago truncatula nodules. New Phytol. 230, 290-303, doi:10.1111/nph.17215 (2021).

64. Capoen, W., Goormachtig, S., De Rycke, R., Schroeyers, K. \& Holsters, M. SrSymRK, a plant receptor essential for symbiosome formation. Proc Natl Acad Sci U S A 102, 10369-10374, doi:10.1073/pnas.0504250102 (2005).

65. Mendoza-Suárez, M., Andersen, S. U., Poole, P. S. \& Sánchez-Cañizares, C. Competition, nodule occupancy, and persistence of inoculant strains: Key Factors in the in the rhizobium-legume symbioses. Front. Plant Sci. 12, 690567, doi:10.3389/fpls.2021.690567 (2021).

66. Bovin, S. \& Lepetit, M. Partner preference in the legume-rhizobia symbiosis and impact on legume inoculation strategies. Advances in Botanical Research, 94, 323-348, doi:10.1016/bs.abr.2019.09.016 (2020).

67. Younginger, B. S. \& Friesen, M. L. Connecting signals and benefits through partner choice in plantmicrobe interactions. FEMS Microbiol. Lett. 366, doi:10.1093/femsle/fnz217 (2019).

68. Van den Bergh, B., Swings, T., Fauvart, M. \& Michiels, J. Experimental design, population dynamics, and diversity in microbial experimental evolution. Microbiol. Mol. Biol. Rev. 82, doi:10.1128/MMBR.00008-18 (2018). 
69. Couce, A. \& Tenaillon, O. A. The rule of declining adaptability in microbial evolution experiments. Front. Genet. 6, 99, doi:10.3389/fgene.2015.00099 (2015).

70. Maddamsetti, R., Lenski, R. E. \& Barrick, J. E. Adaptation, clonal interference, and frequencydependent interactions in a long-term evolution experiment with Escherichia coli. Genetics 200, 619-631, doi:10.1534/genetics.115.176677 (2015).

71. Neher, R. Genetic draft, selective interference, and population genetics of rapid adaptation. Ann. Rev. Ecol. Evol. Syst. 44, 195-215, doi:10.1146/annurev-ecolsys-110512-135920 (2013).

72. Katsnelson, M. I., Wolf, Y. I. \& Koonin, E. V. On the feasibility of saltational evolution. Proc. Natl. Acad. Sci. U. S. A. 116, 21068-21075, doi:10.1073/pnas.1909031116 (2019).

73. Beringer. J. E. R factor transfer in Rhizobium leguminosarum. J. Gen. Microbiol. 84, 188-198, doi: 10.1099/00221287-84-1-188 (1974)

74. Boucher, C. A., Barberis, P. A., Trigalet, A. P. \& Demery, D. A. Transposon mutagenesis of Pseudomonas solanacearum isolation of Tn5-induced avirulent mutants. J. Gen. Microbiol. 131, 2449-2457 (1985).

75. Plener, L., Manfredi, P., Valls, M. \& Genin, S. PrhG, a transcriptional regulator responding to growth conditions, is involved in the control of the type III secretion system regulon in Ralstonia solanacearum. J. Bacteriol. 192, 1011-1019, doi:10.1128/jb.01189-09 (2010).

76. Bertani, G. Studies on lysogenesis. I. The mode of phage liberation by lysogenic Escherichia coli. J. Bacteriol. 62, 293-300, doi:10.1128/jb.62.3.293-300.1951 (1951).

77. Deatherage, D. E. \& Barrick, J. E. Identification of mutations in laboratory-evolved microbes from next-generation sequencing data using breseq. Methods Mol. Biol. 1151, 165-188, doi:10.1007/978-1-4939-0554-6_12 (2014).

78. Robinson, J. T. et al. Integrative genomics viewer. Nat. Biotechnol. 29, 24-26, doi:10.1038/nbt.1754 (2011).

79. Vallenet, D. et al. MicroScope: an integrated platform for the annotation and exploration of microbial gene functions through genomic, pangenomic and metabolic comparative analysis. Nucleic Acids Res 48, D579-D589, doi:10.1093/nar/gkz926 (2020).

80. R Core Team (2019). R: A language and environment for statistical computing. R Foundation for Statistical (2019).

81. Noble, R. ggmuller: Create Muller plots of evolutionary dynamics. R package version 0.5.3. doi:10.5281/zenodo.591304 (2019).

82. Dalia, A. B., McDonough, E. \& Camilli, A. Multiplex genome editing by natural transformation. Proc. Natl. Acad. Sci. U. S. A. 111, 8937-8942, doi:10.1073/pnas.1406478111 (2014).

83. Chen, W. M. et al. Legume symbiotic nitrogen fixation by beta-proteobacteria is widespread in nature. J. Bacteriol. 185, 7266-7272, doi:10.1128/jb.185.24.7266-7272.2003 (2003). 
84. Fahraeus, G. The infection of Clover root hairs by nodule bacteria studied by a simple glass slide technique. J. Gen. Microbiol. 16, 374-\& (1957).

85. Jensen, H. L. Nitrogen fixation in leguminous plants. I. General characters of root nodule bacteria isolated from species of Medicago and Trifolium in Australia. Proc. Int. Soc. N.S.W. 66, 68-108 (1942).

\section{Figure legend}

Fig. 1 | Evolution of the symbiotic properties of Ralstonia clones along evolution cycles.

a, Left: overview of the evolution cycles showing the symbiotic steps determining bacterial fitness (nodulation competitiveness and within-host proliferation) and human intervention (nodule harvest, grinding and re-inoculation). Nodule colors reflect bacterial genotypes: blue represents the ancestor, and red represents a mutant with increased fitness. Right: schematic representation of the main phenotypic measurements performed in this work, where the symbiotic phenotypes of one evolved strain can be compared to those of a reference strain (e.g. the reference symbiont $C$. taiwanensis). Competitive indexes for in planta fitness are calculated as the ratio of evolved vs. reference clones in nodule bacterial populations normalized by the ratio of strains in the inoculum. Competitive indexes for nodulation competitiveness are calculated as the ratio of nodules formed by each strain normalized by the inoculum ratio. Within-host proliferation is measured in independent single inoculations of each strain. b-d, Relative in planta fitness (b), nodulation competitiveness (c) and within-host proliferation (d) of nodulating ancestors (cycle 0) and evolved clones isolated from cycles 16 and 35 were compared to $C$. taiwanensis. Values correspond to means \pm standard deviations. Data were obtained from at least three independent experiments. For each experiment, nodules were harvested from 10 plants (b), 20 plants $(\mathbf{c})$ and 6 plants $(\mathbf{d})$. The sample size $(n)$ is equal to $n=3(\mathbf{b}, \mathbf{c})$ or comprised between $n=15-18(\mathbf{d})$. Raw data are available in Supplementary Table 2. cfu: colony-forming units.

\section{Figure 2. Dynamics of molecular evolution}

Allele frequency trajectories of mutations that attained a frequency of $30 \%$ in at least one population of the $B, F, G, K$ and $M$ lineages $(\mathbf{a}, \mathbf{b}, \mathbf{c}, \mathbf{d}, \mathbf{e})$. Mutations with similar trajectories were clustered in cohorts and represented by different colors. For simplicity, mutations travelling alone were also called cohorts.

Figure 3. Symbiotic phenotypes of reconstructed mutants and evolved clones from lineages B and G. a,d, In planta relative fitness of evolved clones carrying reconstructed mutations from fixed mutational cohorts. Evolved clones in which mutations were reconstructed are indicated on the left of the graph. Horizontal lines separate the different mutational cohorts. Cohort number and the number of tested 
mutations from the cohort on the total number of mutations present in the cohort are indicated in brackets. Competitive indexes $(\mathrm{Cl})$ were calculated as the ratio of the mutant strain on the isogenic parental strain in bacterial nodule populations normalized by the inoculum ratio. GFP/mCherry correspond to control co-inoculation experiments of strains derived from the same evolved clone labelled with different fluorophore either GFP or mCherry. b,e, Nodulation competitiveness effect of adaptive mutants. $\mathrm{Cl}$ were calculated as the ratio of the number of nodules formed by the mutant strain on the number of nodules formed by the isogenic parental strain normalized by the inoculum ratio. a,b,d,e, vertical segments correspond to mean values of $\mathrm{Cl}$. Red segments indicate significantly beneficial mutations, gray segments indicate neutral mutations and blue segments indicate significantly deleterious mutations $(P<0.05, t$-test). $\mathbf{c}, \mathbf{f}$, Within-host proliferation (number of bacteria per nodule) of adaptive mutants and the corresponding isogenic parental clones. Rectangles span the first quartiles to the third quartiles, bold segments inside the rectangle show the median, unfiled circles represent outliers, whiskers above and below each box show the minimum and the maximum in the absence of suspected outlying data. Red fillings indicate the mutations that significantly improved within-host proliferation compared to the parental evolved clone ( $P<0.05$, Wilcoxon test). $\mathbf{a}, \mathbf{b}, \mathbf{c}, \mathbf{d}, \mathbf{e}, \mathbf{f}$, Data were obtained from 3 to 5 independent experiments. For each experiment, nodules were harvested from 10 plants $(\mathbf{a}, \mathbf{d}), 20$ plants $(\mathbf{b}, \mathbf{e})$ and 6 plants $(\mathbf{c}, \mathbf{f})$. The sample size $(\mathrm{n})$ is comprised between $n=3-5(\mathbf{a}, \mathbf{d}), n=3-4(\mathbf{b}, \mathbf{e})$ and $n=15-24(\mathbf{c}, \mathbf{f})$. Raw data are available in Supplementary Table 9.

\section{Figure 4. Relative strength of selection for nodulation competitiveness and proliferation}

a, Median fitness trajectories of 100 simulated populations evolving under different sizes of nodulation bottleneck (10 to 3000 nodules). Points indicate fitness values at cycles $0,10,20,30,40$ and 50 . Dotted grey lines represent iso-fitness lines (defined as the product of nodulation competitiveness and proliferation) used to delineate 3 fitness domains: Low (L; fitness $\left.<10^{-5}\right)$, Mid $\left(\mathrm{M} ; 10^{-5}<\right.$ fitness $\left.<10^{-3}\right)$, and High $\left(\mathrm{H}\right.$; fitness $\left.>10^{-3}\right)$. The black dotted line represents the diagonal, along which population would improve both phenotypic traits equally well. $\mathbf{b}$, Fold effect (nodulation competitiveness effect divided by proliferation effect) of mutations that reached a frequency of at least $30 \%$ in simulated populations. Color shading indicate the fitness domain (Low, Mid or High; shown in panel a) in which each group of mutations arose. Note that fold effects of mutations are indicated even when median fitness trajectories do not reach the High fitness domain (e.g. 10 and 30 nodule lines in a) because some individual replicate simulations (shown in Supplementary Figures) do reach this fitness domain. Figures $\mathbf{c}$ and $\mathbf{d}$ are built following the same principles than $\mathbf{a}$ and $\mathbf{b}$, but explore the effect of differences in initial fitnesses. Note that the dashed diagonal (in b) or horizontal (in d) lines represent the expected evolutionary trajectories when initial fitnesses are unequal, and upon the null hypothesis that adaptation would proceed proportionally to the differences between the ancestral and the theoretical 
optimum values for each phenotypic component. Figures $\mathbf{e}$ and $\mathbf{f}$ are as $\mathbf{a}$ and $\mathbf{b}$ but explore a situation where the nodulation bottleneck occurs after bacterial clonal proliferation.

\section{Tables}

Table 1. Symbiotic phenotypes of beneficial mutations improving in planta fitness.

\section{Acknowledgements}

We are grateful to A. Carlier and F. Roux for careful reading of the manuscript, to O. Tenaillon for helpful discussions on the project, to $\mathrm{O}$. Tenaillon and $\mathrm{H}$. Le Nagard for sharing their genetic algorithm script, and to Ludovic Legrand for archiving sequencing data and their associated metadata. We acknowledge the Genotoul bioinformatics platform Toulouse Occitanie (Bioinfo Genotoul, https://doi.org/10.15454/1.5572369328961167E12) for providing computing and storage resources. G.G.D.d.M was supported by a fellowship from the French Ministère de l'Enseignement Supérieur, de la Recherche et de I'Innovation (MESRI). P.R. received funding from the EU in the framework of the Marie-Curie FP7 COFUND People Programme, through the award of an AgreenSkills+ fellowship (under grant agreement $n^{\circ} 609398$ ) and from the European Union's Horizon 2020 research and innovation programme under the Marie Skłodowska-Curie grant agreement $N^{\circ} 845838$.

This study was supported by the Fédération de Recherche Agrobiosciences, Interactions et Biodiversité, the French National Research Agency (ANR-16-CE20-0011-01 and ANR-21-CE02-0019-01) and the "Laboratoires d'Excellence (LABEX)" TULIP (ANR-10-LABX-41)" and the "École Universitaire de Recherche (EUR)" TULIP-GS (ANR-18-EURE-0019).

\section{Authors contributions}

C.M.-B, D.C. and P.R. designed the project. G.G.D.M. performed most experiments and analyze the data. S.M., N.G., A.-C.C., D.C. and P.R. performed experiments. J.-B.F. and P.R. performed computer simulations. M.M., T.B. and S.V. performed NGS sequencing at GeT-PlaGe. D.R. analyzed mutations in clones and deposit data in the microScope platform. D.C. and P.R. supervised experimental work and data analysis. C.B.-M., J.-B.F and P.R. acquired funding for the project. G.G.D.M., J.B-F., D.C. and P.R. wrote the manuscript, which was approved by all authors.

\section{Competing interests}

The authors declare no competing interests.

\section{Extended data}



serial cycles of plant (Mimosa pudica) inoculation-isolation of nodule bacteria.

893 The 5 bacterial lineages, B, F, G, K, and $\mathrm{M}$ lines were independently derived from the spontaneous nodulating mutants either CBM212, or CBM349, or CBM356. At each evolution cycle, bacterial nodule populations were isolated either 21 days (black lines) or 42 days (blue lines) after plant inoculation and partly directly re-inoculated to new plants to initiate the next cycle. At each cycle, one individual clone representative of the bacterial population was isolated and stored. Sequenced populations and clones are indicated. The number of nodules harvested at each evolution cycle is reported in Supplementary Table 1.

Extended Data Fig. 2. Evolution of population composition over time in lineages B and G.

Muller plots representing the relative frequency of each genotype in lineages $B(a)$ and $G(b)$ for each sequenced population (uneven cycles). Each color represent a different genotype, and its relative frequency is shown as the vertical area at each sequenced time point. The genotypes containing cohorts for which adaptive mutations were identified experimentally are indicated. For each cohort, non-adaptive or deleterious mutations are shown in black, adaptive mutations are in red.

Extended Data Fig. 3. Survival of reconstructed mutants from lineages B and $G$ in the Jensen medium and rhizosphere.

Survival of evolved clones carrying reconstructed adaptive mutations from fixed mutational cohorts in the Jensen medium (a) and in rhizosphere (b). Competitive indexes $(\mathrm{Cl})$ were calculated as the ratio of the mutant strain on the isogenic parental strain in Jensen medium populations normalized by the inoculum ratio. Red points correspond to mean values of $\mathrm{Cl}$. Rectangles span the first quartiles to the third quartiles, bold segments inside the rectangle show the median. Red stars beside strain names indicate the mutations that significantly improved survival in the rhizosphere compared to the parental evolved clone ( $P<0.05$, Wilcoxon test with Benjamini-Hochberg correction). Data were obtained from at least 3-5 independent experiments for each comparison and the sample size ( $n$ ) is comprised between $n=9-14$. Raw data are available in Supplementary Table 10.

Extended Data Table 1. Summary of detected mutations in the five lineages

921 Extended Data Table 2. Analysis of genetic parallelism in the five lineages.

922 Extended Data Table 3. Global analysis of mutational cohorts

923 Extended Data Table 4. Neutral and deleterious reconstructed mutations

924 Extended Data Table 5. Frequencies of usage of codons modified by adaptive synonymous mutations 
928 Supplementary Table 1. Number of nodules harvested at each evolution cycle.

929 Supplementary Table 2. Raw data obtained for the phenotypic characterization of Ralstonia evolved clones compared to $C$. taiwanensis.

931 Supplementary Table 3. Coverage depth for whole-population sequencing

932 Supplementary Table 4. List of mutations detected in evolved populations of the B lineage.

933 Supplementary Table 5. List of mutations detected in evolved populations of the F lineage.

934 Supplementary Table 6. List of mutations detected in evolved populations of the G lineage.

935 Supplementary Table 7. List of mutations detected in evolved populations of the K lineage.

936 Supplementary Table 8. List of mutations detected in evolved populations of the M lineage.

937 Supplementary Table 9. Raw data obtained for the in planta phenotypic characterization of

938 reconstructed mutants compared to their respective isogenic parental strain.

939 Supplementary Table 10. Raw data obtained for the ex planta phenotypic characterization of

940 reconstructed mutants compared to their respective isogenic parental strain.

941 Supplementary Table 11. Strains and plasmids used in this study.

942 Supplementary Table 12. List of mutations detected in evolved clones of the B lineage.

943 Supplementary Table 13. List of mutations detected in evolved clones of the F lineage.

944 Supplementary Table 14. List of mutations detected in evolved clones of the $\mathrm{G}$ lineage.

945 Supplementary Table 15. List of mutations detected in evolved clones of the $\mathrm{K}$ lineage.

946 Supplementary Table 16. List of mutations detected in evolved clones of the $M$ lineage.

947 Supplementary Table 17. Oligonucleotides used in this study.

948 Supplementary Table 18. Parameter values used in computer simulations

949

950 Supplementary Information. Model framework and supplementary simulation results

951

952

Correspondence and requests for materials should be addressed to P.R. or D.C. 
bioRxiv preprint doi: https://doi.org/10.1101/2022.03.03.482760; this version posted March 4, 2022. The copyright holder for this preprint

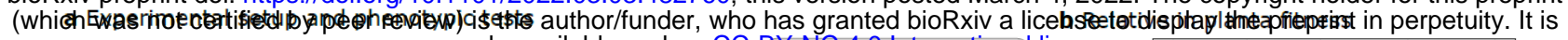
made available under aCC-BY-NGetato Interinational license.
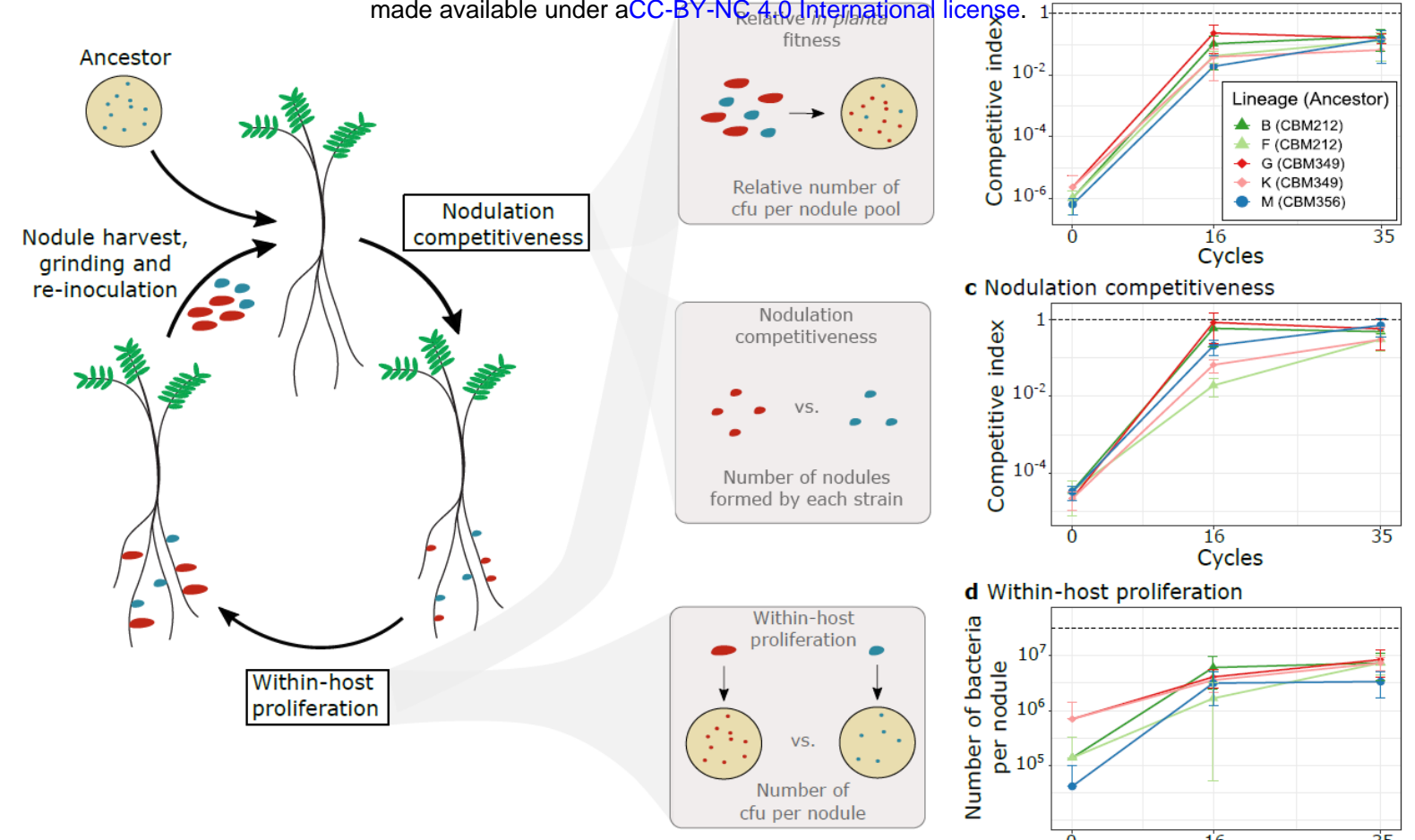

c Nodulation competitiveness

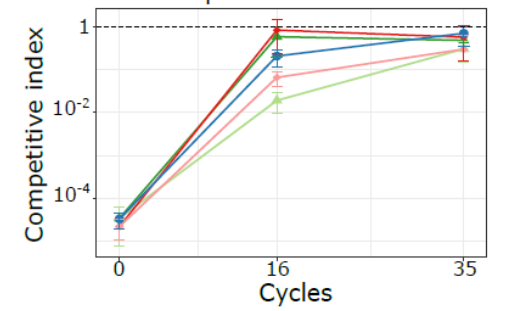

d Within-host proliferation

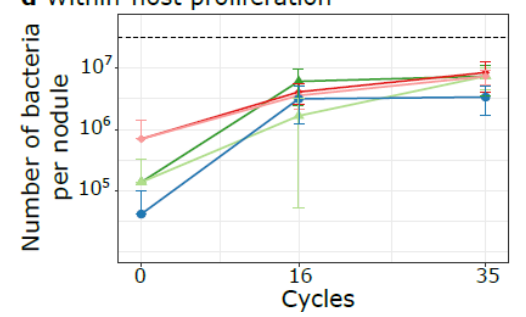

Fig. 1 | Evolution of the symbiotic properties of Ralstonia clones along evolution cycles.

a, Left: overview of the evolution cycles showing the symbiotic steps determining bacterial fitness (nodulation competitiveness and within-host proliferation) and human intervention (nodule harvest, grinding and re-inoculation). Nodule colors reflect bacterial genotypes: blue represents the ancestor, and red represents a mutant with increased fitness. Right: schematic representation of the main phenotypic measurements performed in this work, where the symbiotic phenotypes of one evolved strain can be compared to those of a reference strain (e.g. the reference symbiont $C$. taiwanensis). Competitive indexes for in planta fitness are calculated as the ratio of evolved $v s$. reference clones in nodule bacterial populations normalized by the ratio of strains in the inoculum. Competitive indexes for nodulation competitiveness are calculated as the ratio of nodules formed by each strain normalized by the inoculum ratio. Within-host proliferation is measured in independent single inoculations of each strain. b-d, Relative in planta fitness (b), nodulation competitiveness (c) and within-host proliferation (d) of nodulating ancestors (cycle 0 ) and evolved clones isolated from cycles 16 and 35 were compared to $C$. taiwanensis. Values correspond to means \pm standard deviations. Data were obtained from at least three independent experiments. For each experiment, nodules were harvested from 10 plants (b), 20 plants $(\mathbf{c})$ and 6 plants $(\mathbf{d})$. The sample size $(n)$ is equal to $n=3(\mathbf{b}, \mathbf{c})$ or comprised between $\mathrm{n}=15-18$ (d). Raw data are available in Supplementary Table 2. cfu: colony-forming units. 
bioRxiv preprint doi: https://doi.org/10.1101/2022.03.03.482760; this version posted March 4, 2022. The copyright holder for this preprint (which was not certified by peer review) is the author/funder, who has granted bioRxiv a license to display the preprint in perpetuity. It is made available under aCC-BY-NC 4.0 International license.

a Lineage $B$

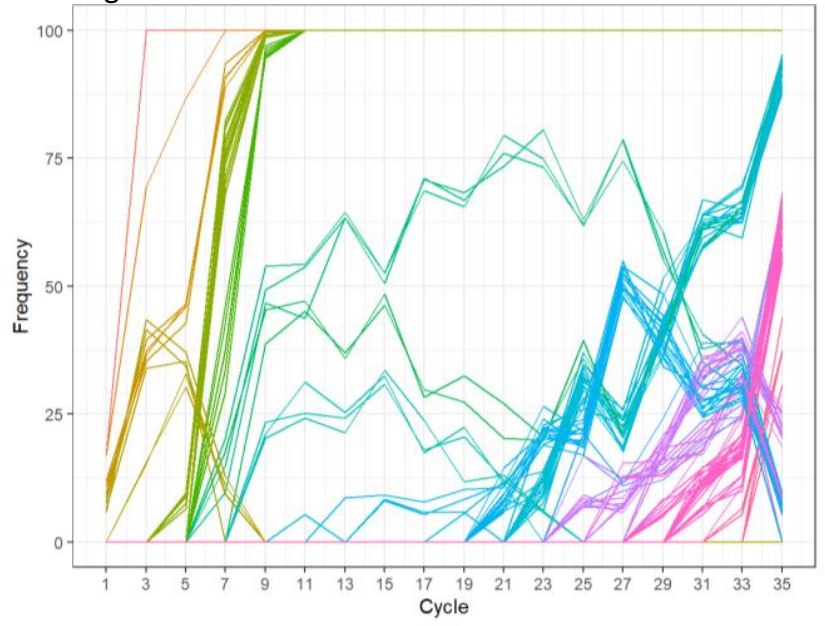

b Lineage $\mathrm{F}$

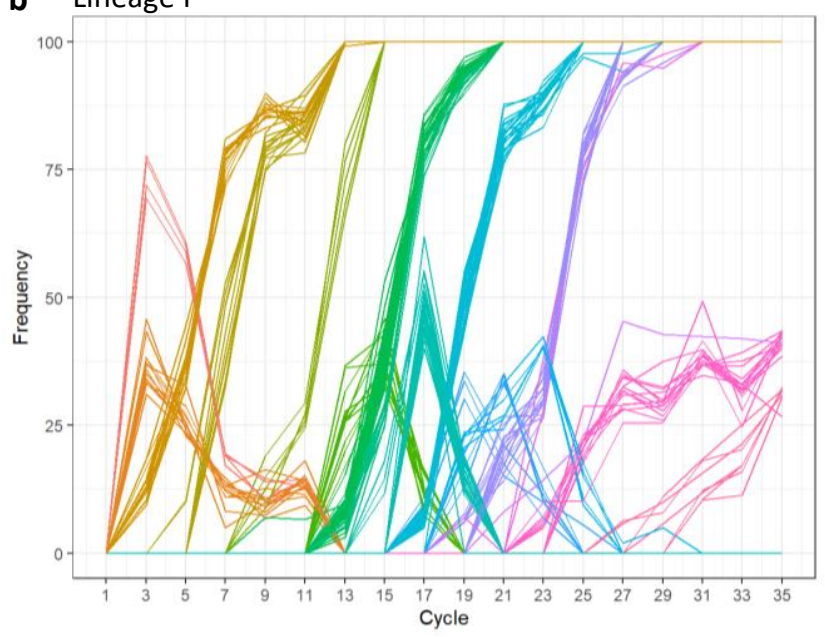

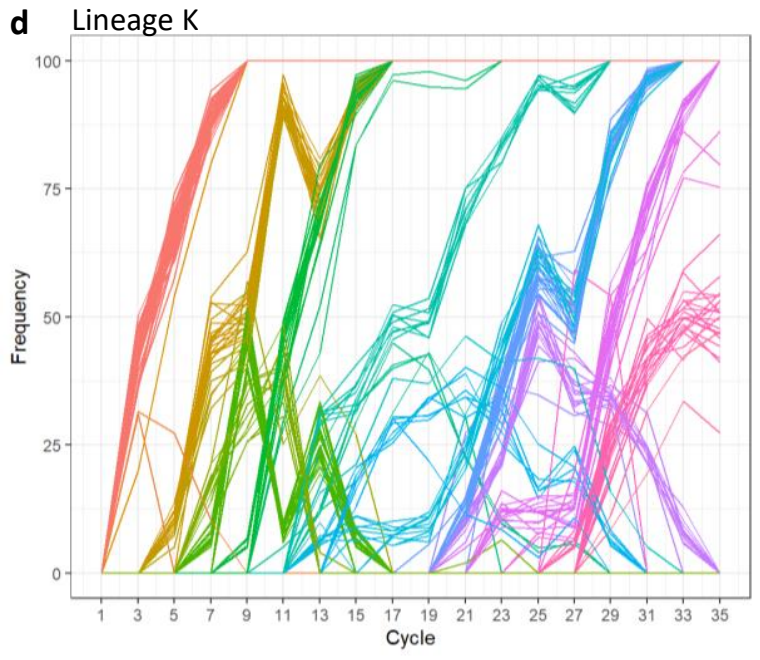

e Lineage $M$
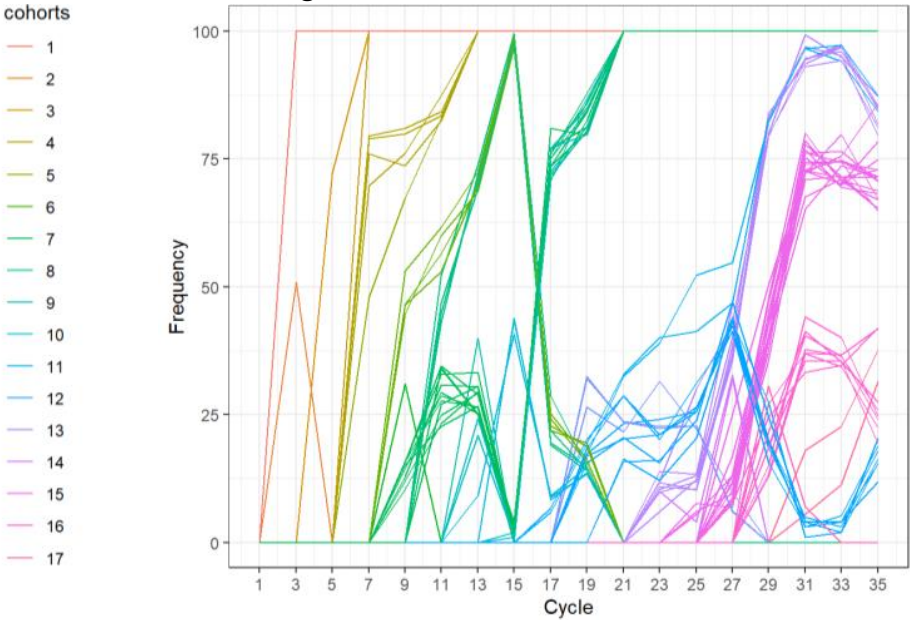

c Lineage G

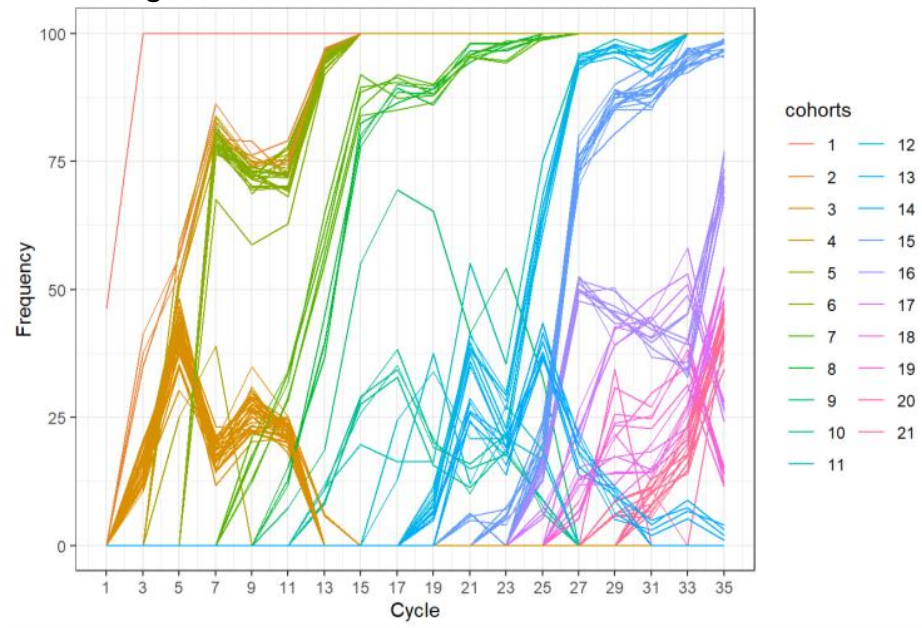

Fig. 2 | Dynamics of molecular evolution. Allele frequency trajectories of mutations that attained a frequency of $30 \%$ in at least one population of the $B, F, G, K$ and $M$ lineages $(\mathbf{a}, \mathbf{b}, \mathbf{c}, \mathbf{d}, \mathbf{e})$. Mutations with similar trajectories were clustered in cohorts and represented by different colors. For simplicity, mutations travelling alone were also called cohorts. 
bioRxiv preprint doi: https://doi.org/10.1101/2022.03.03.482760; this version posted March 4, 2022. The copyright holder for this preprint (which was not certified by peer review) is the author/funder, who has granted bioRxiv a license to display the preprint in perpetuity. It is made available under aCC-BY-NC 4.0 International license.

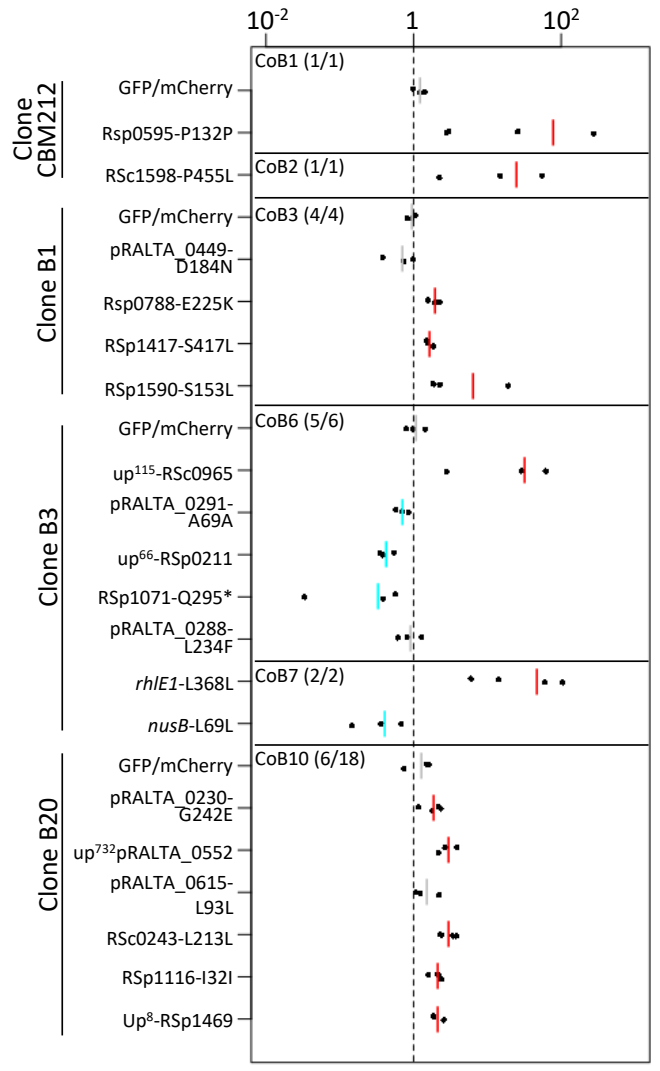

d

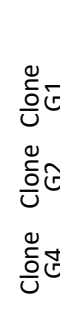

$\stackrel{\frac{0}{U}}{\frac{\Xi}{U}}$

Relative in planta fitness

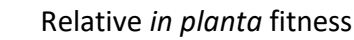

b

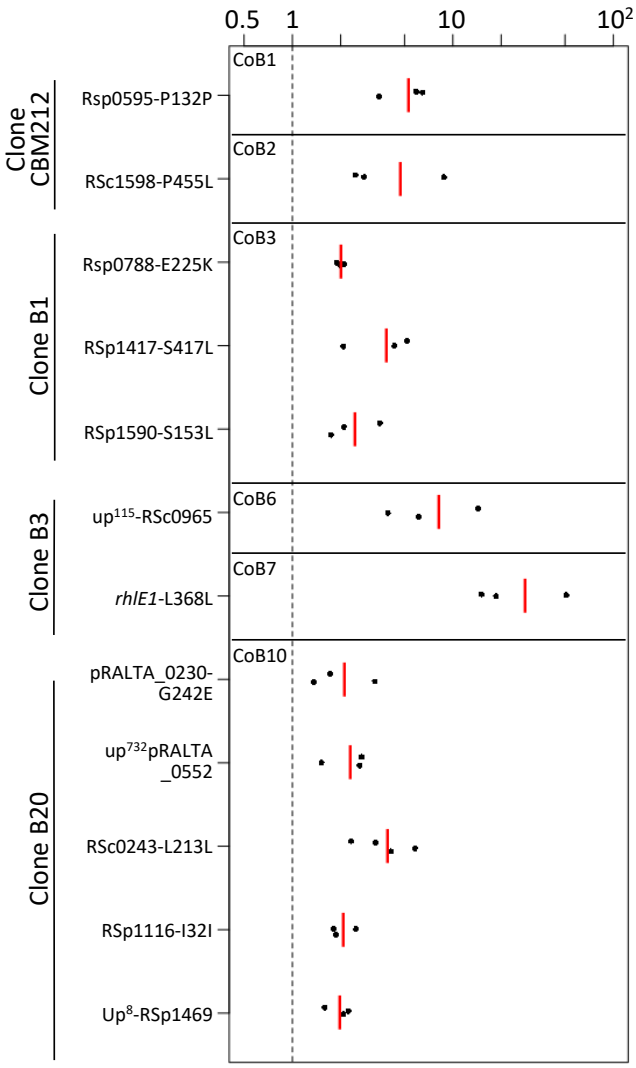

e

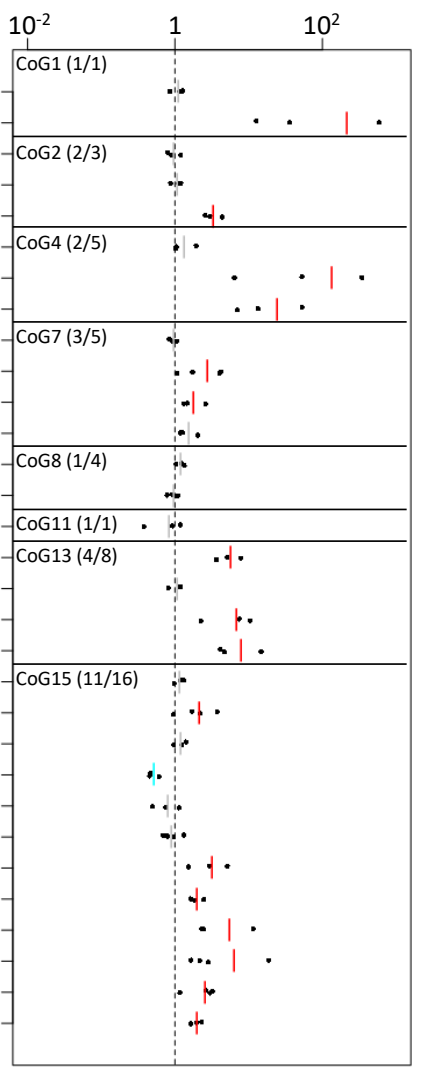

Nodulation competitiveness

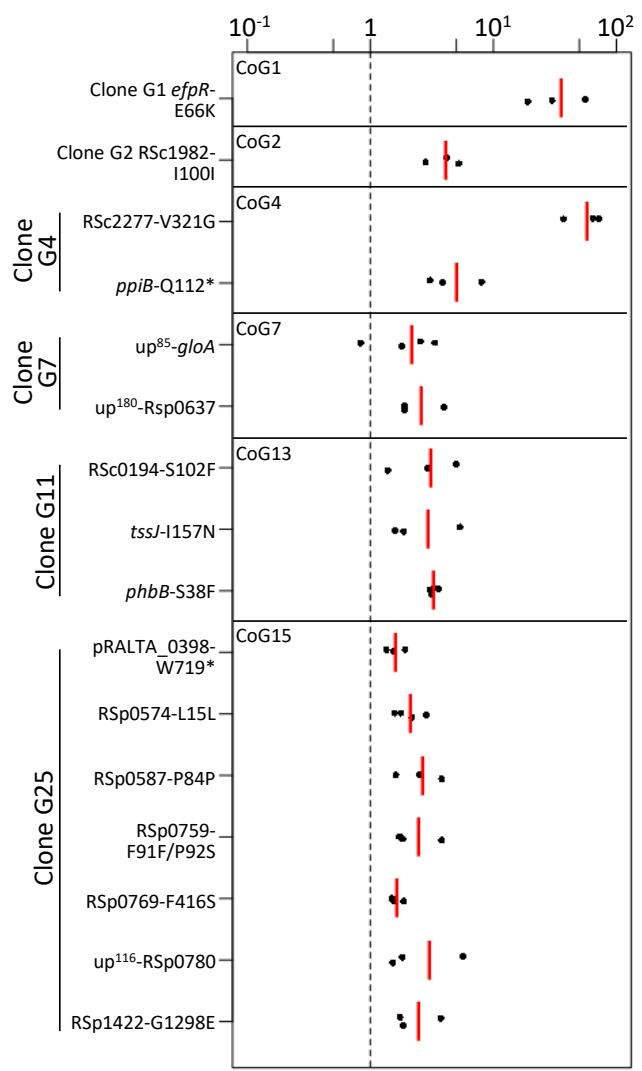

c

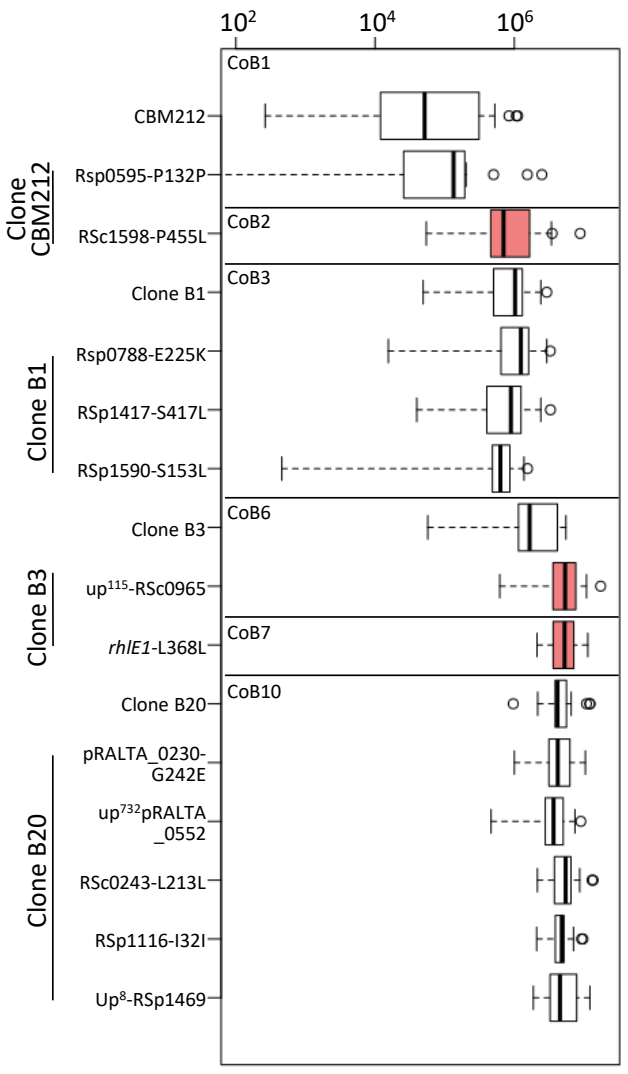

f
Number of bacteria per nodule

Number of bacteria per nodule

CoG2


bioRxiv preprint doi: https://doi.org/10.1101/2022 03 03.482760; this version posted March 4, 2022. The copyright holder for this preprint (which was not certified by peer review) is the author/funder, who has granted bioRxiv a license to display the preprint in perpetuity. It is made available under aCC-BY-NC 4.0 International license.

Fig. 3| Symbiotic phenotypes of reconstructed mutants and evolved clones from lineages B and G.

a,d, In planta relative fitness of evolved clones carrying reconstructed mutations from fixed mutational cohorts. Evolved clones in which mutations were reconstructed are indicated on the left of the graph. Horizontal lines separate the different mutational cohorts. Cohort number and the number of tested mutations from the cohort on the total number of mutations present in the cohort are indicated in brackets. Competitive indexes $(\mathrm{Cl})$ were calculated as the ratio of the mutant strain on the isogenic parental strain in bacterial nodule populations normalized by the inoculum ratio. GFP/mCherry correspond to control co-inoculation experiments of strains derived from the same evolved clone labelled with different fluorophore either GFP or mCherry. b,e, Nodulation competitiveness effect of adaptive mutants. $\mathrm{Cl}$ were calculated as the ratio of the number of nodules formed by the mutant strain on the number of nodules formed by the isogenic parental strain normalized by the inoculum ratio. $\mathbf{a}, \mathbf{b}, \mathbf{d}, \mathbf{e}$, vertical segments correspond to mean values of $\mathrm{Cl}$. Red segments indicate significantly beneficial mutations, gray segments indicate neutral mutations and blue segments indicate significantly deleterious mutations $(P<0.05, t$-test). c,f, Distribution of within-host proliferation values (number of bacteria per nodule) of adaptive mutants and the corresponding isogenic parental clones. Rectangles span the first quartiles to the third quartiles, bold segments inside the rectangle show the median, unfiled circles represent outliers, whiskers above and below each box show the minimum and the maximum in the absence of suspected outlying data. Red stars indicate the mutations that significantly improved infectivity compared to the parental evolved clone ( $P<0.05$, Wilcoxon test). a, b, $, \mathbf{c}, \mathbf{d}, \mathbf{e}, \mathbf{f}$, Data were obtained from 3 to 5 independent experiments. For each experiment, nodules were harvested from 10 plants $(\mathbf{a}, \mathbf{d}), 20$ plants $(\mathbf{b}, \mathbf{e})$ and 6 plants $(\mathbf{c}, \mathbf{f})$. The sample size $(n)$ is comprised between $n=3-5(\mathbf{a}, \mathbf{d}), n=3-4(\mathbf{b}, \mathbf{e})$ and $n=15-24(\mathbf{c}, \mathbf{f})$. Raw data are available in Supplementary Table 9. 
a

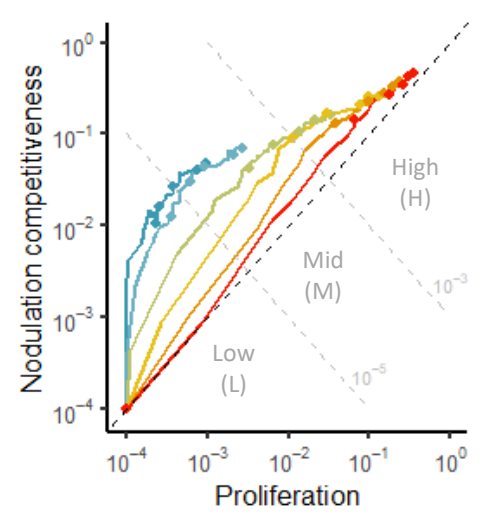

c

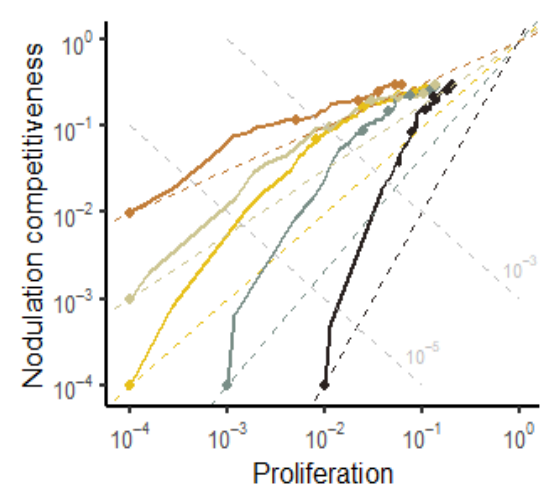

Log10 starting fitness (Nod/Pro)

$\rightarrow-2 /-4$

$\rightarrow-3 / 4$

$\rightarrow-4 /-4$

$\rightarrow-4 /-3$

$\rightarrow-4 /-2$

e

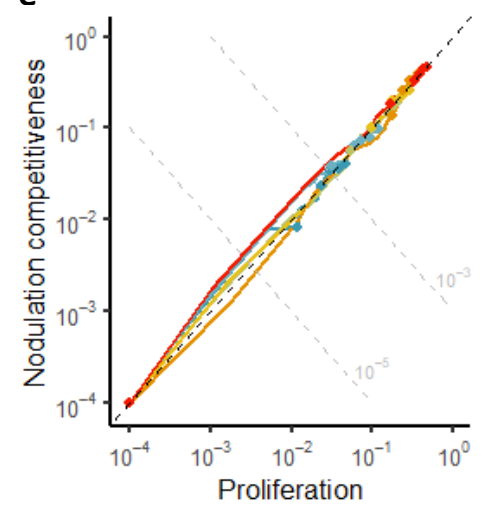

b

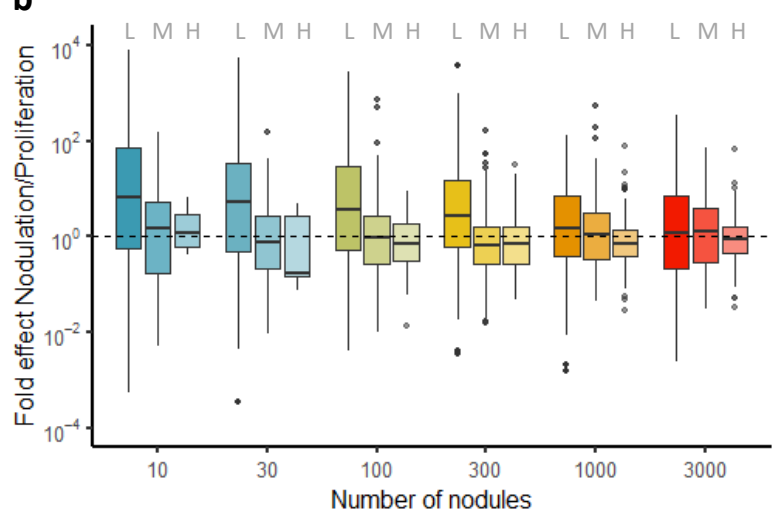

d
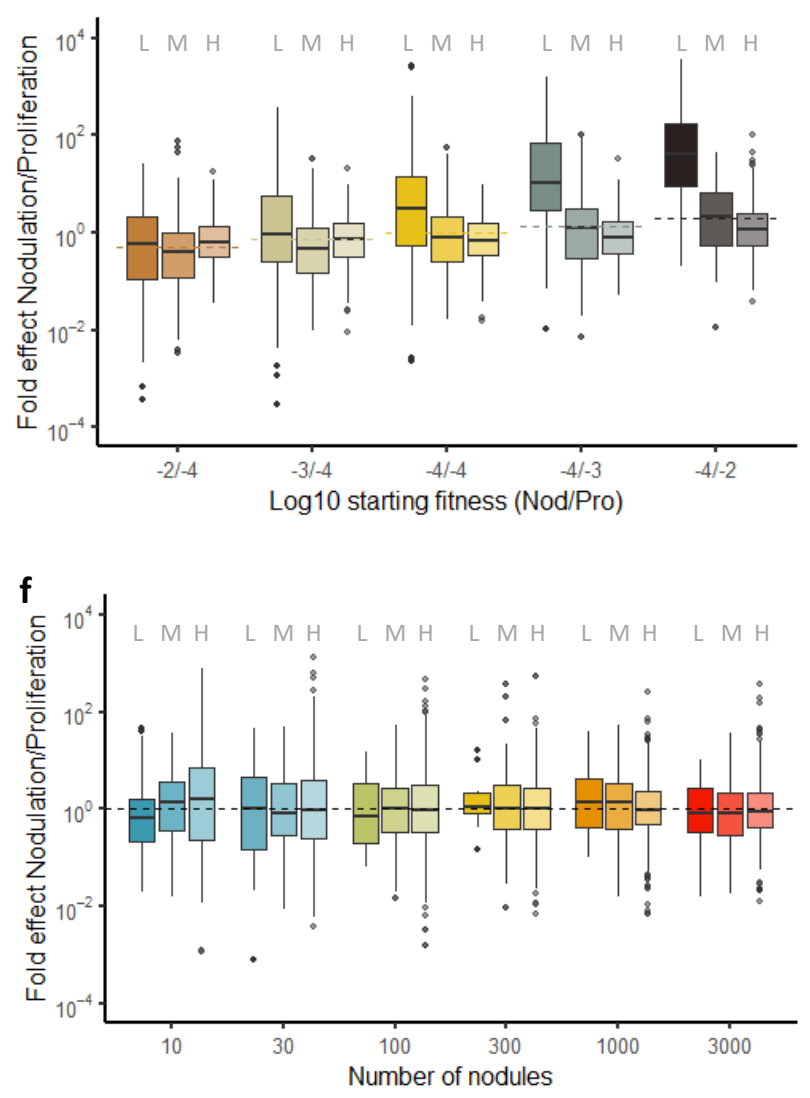

Fig. 4 | Relative strength of selection for nodulation competitiveness and proliferation

a,c,e, Median fitness trajectories of 100 simulated populations evolving under different sizes of nodulation bottleneck (10 to 3000 nodules). Points indicate fitness values at cycles $0,10,20,30,40$ and 50. Dotted grey lines represent isofitness lines (defined as the product of nodulation competitiveness and proliferation) used to delineate 3 fitness domains: Low $\left(\mathrm{L}\right.$; fitness $\left.<10^{-5}\right)$, Mid $\left(\mathrm{M} ; 10^{-5}<\right.$ fitness $\left.<10^{-3}\right)$, and High $\left(\mathrm{H}\right.$; fitness $\left.>10^{-3}\right)$. The black dotted line represents the diagonal, along which population would improve both phenotypic traits equally well. b,d,f, Fold effect (nodulation competitiveness effect divided by proliferation effect) of mutations that reached a frequency of at least $30 \%$ in simulated populations. Color shading indicate the fitness domain (Low, Mid or High) in which each group of mutations arose. Note that fold effects of mutations are indicated even when median fitness trajectories do not reach the High fitness domain (e.g. 10 and 30 nodule lines in a) because some individual replicate simulations (shown in Supplementary Figures) do reach this fitness domain. The effect of different parameters on adaptive trajectories was tested: the size of nodulation bottleneck $(\mathbf{a}, \mathbf{b})$, the initial fitness values of the ancestor $(\mathbf{c}, \mathbf{d})$, and the size of nodulation bottleneck under a scenario where the nodulation bottleneck occurs after bacterial clonal proliferation (e, $\mathbf{f})$. 


\section{Mean Cl for Mean gain}

plant culture Mean Cl for Mean Cl for

\begin{tabular}{|c|c|c|c|c|c|c|c|c|c|c|c|c|c|c|}
\hline Lineage & Gene ID ${ }^{a}$ & $\begin{array}{l}\text { Gene } \\
\text { name }\end{array}$ & Mutation & $\begin{array}{c}\text { Type of } \\
\text { mutation }\end{array}$ & Description & Cohort & $\begin{array}{l}\text { Mean } \mathrm{Cl} \text { for in } \\
\text { planta fitness }\end{array}$ & $\begin{array}{c}\text { medium } \\
\text { colonization }^{\mathrm{d}}\end{array}$ & $\begin{array}{l}\text { rhizosphere } \\
\text { colonization }^{\text {d }}\end{array}$ & $\begin{array}{c}\text { nodulation } \\
\text { competitiveness }^{\mathrm{c}}\end{array}$ & $\begin{array}{c}\text { host } \\
\text { proliferation }\end{array}$ & $\begin{array}{c}\text { Symbiotic } \\
\text { phenotypes }^{\mathrm{f}}\end{array}$ & G score ${ }^{\mathrm{g}}$ & $\begin{array}{c}\text { Adjusted } \\
P \text { value }^{\mathrm{h}}\end{array}$ \\
\hline \multirow[t]{12}{*}{ B } & RSp0595 & \multirow{12}{*}{ rhlE1 } & P132P & Syn & Hypothetical protein & COB1 & 77 & 1 & 1 & 5.2 & 1.6 & Nod+ & 2.80 & 1 \\
\hline & RSc1598 & & P455L & Nonsyn & Transmembrane sensor histidine kinase & Сов2 & 24.2 & 1 & 1.4 & 4.7 & 6.4 & Nod+ Prot & 1.04 & 1 \\
\hline & RSp0788 & & E225K & Nonsyn & Carbamoyl-transferase & COB3 & 1.9 & 1 & 1.1 & 2 & 1.2 & Nod+ & -0.09 & 1 \\
\hline & RSp1417 & & S417L & Nonsyn & Multidrug efflux transmembrane protein & COB3 & 1.6 & 0.8 & 1.4 & 3.9 & 0.9 & Nodt & 12.47 & $2.58 \mathrm{E}-04$ \\
\hline & RSp1590 & & S153L & Nonsyn & FAD-dependent oxireductase & СОВ3 & 6.3 & 1 & 0.9 & 2.4 & 0.6 & Nod+ & 4.10 & 1 \\
\hline & up $^{115}-$ RSc0965 & & G/A & Intergenic & Unknown function, negatively regulates efpR expression & COB6 & 31.1 & 0.9 & 2.9 & 8.2 & 2.6 & Rhizo+ Nod+ Prot & nd & nd \\
\hline & Rsc0539 & & L368L & Syn & ATP-dependent RNA helicase protein & COB7 & 46.2 & 1.1 & 4 & 28.2 & 2.4 & Rhizo+ Nod+ Prot & 2.88 & 1 \\
\hline & RSc0243 & & L213L & Syn & LysR-type transcription regulator protein & COB10 & 3 & 1.3 & 1.4 & 3.9 & 1.1 & Rhizo+ Nod+ & 1.16 & 1 \\
\hline & Rsp1116 & & $\begin{array}{l}1321 \\
\mathrm{~L} 33 \mathrm{~F}\end{array}$ & $\begin{array}{l}\text { Syn } \\
\text { Nonsyn }\end{array}$ & Putative low specificity L-threonine aldolase & COB10 & 2.1 & 1.2 & 1.2 & 2.1 & 0.9 & Nodt & 0.84 & 1 \\
\hline & $u p^{8}-\mathrm{RSp} 1469$ & & $\mathrm{C} / \mathrm{T}$ & Intergenic & Putative tyrosine specific protein phosphatase & COB10 & 2.1 & 0.9 & 1 & 2 & 1.0 & Nodt & nd & nd \\
\hline & PRALTA_0230 & & G242E & Nonsyn & Putative ATP-dependent DNA helicase & COB10 & 1.5 & 1.5 & 1.5 & 2.1 & 0.9 & Nodt & 6.34 & 1 \\
\hline & PRALTA_0552 & & 11001 & Syn & Pseudogene, putative membrane bound hydrogenase & CoB10 & 2.9 & 1.2 & 1.4 & 2.3 & 0.8 & Nod+ & nd & nd \\
\hline \multirow[t]{16}{*}{ G } & RSc1097 & efpR & E66K & Nonsyn & Transcriptional regulator & CoG1 & 212.1 & 1.3 & 4 & 35 & 3.7 & Rhizo+ Nod+ Prot & 3.17 & 1 \\
\hline & RSc1982 & & 11001 & Syn & DNA methyltransferase & COG2 & 3.3 & 3.1 & 1.3 & 4.0 & 0.7 & Nod+ & 1.44 & 1 \\
\hline & Rsc1164 & \multirow[t]{2}{*}{ ррів } & Q112* & Nonsense & Peptyl-prolyl-cis/trans isomerase & CoG4 & 24.5 & 1 & 1.0 & 5.0 & 1.4 & Nod+ & 2.26 & 1 \\
\hline & RSc2277 & & V321G & Nonsyn & Putative transporter protein & $\operatorname{cog} 4$ & 133.9 & 9.3 & 10.1 & 58 & 2.5 & Nod+ Prot & 36.95 & $4.68 \mathrm{E}-50$ \\
\hline & $\mathrm{up}^{85}$-RSC0520 & \multirow[t]{2}{*}{ gloA } & $\mathrm{T} / \mathrm{C}$ & Intergenic & Lactoylglutathione lyase & COG7 & 2.8 & 1.2 & 1.5 & 2.1 & 1.1 & Nod+ & nd & nd \\
\hline & $\mathrm{up}^{180}-\mathrm{RSp} 0637$ & & $\mathrm{G} / \mathrm{C}$ & Intergenic & Hypothetical protein & CoG7 & 1.8 & 1 & 1.2 & 2.6 & 0.9 & Nodt & nd & nd \\
\hline & RSc0194 & \multirow{10}{*}{$\begin{array}{c}\text { phbB } \\
\text { tssJ }\end{array}$} & S102F & Nonsyn & Zinc-dependent alcohol dehydrogenase & CoG13 & 5.5 & 1.1 & 1.6 & 3.1 & 1.4 & Nod+ & 4.67 & 1 \\
\hline & RSc1633 & & S38F & Nonsyn & Acetoacetyl-CoA reductase & CoG13 & 7.9 & 2 & 2.1 & 3.3 & 1.7 & Rhizo+ Nod+Pro+ & 1.62 & 1 \\
\hline & RSp0741 & & $1157 \mathrm{~N}$ & Nonsyn & Type VI secretion system, lipoprotein TssJ & CoG13 & 6.7 & 1 & 1.1 & 2.9 & 1.7 & Nod+ & 6.99 & 1 \\
\hline & RSp0574 & & L15L & Syn & Hypothetical protein & CoG15 & 3.2 & 1.1 & 1.3 & 2.1 & 1.6 & Nod+Pro+ & 4.36 & 1 \\
\hline & RSp0587 & & P84P & Syn & Putative signal peptide hypothetical protein & CoG15 & 2 & 1.1 & 0.8 & 2.6 & 1.3 & Nodt & 28.74 & $1.04 E-46$ \\
\hline & RSp0759 & & $\begin{array}{l}\text { F91F } \\
\text { P92S }\end{array}$ & $\begin{array}{c}\text { Syn } \\
\text { Nonsyn }\end{array}$ & Putative type VI secretion-associated protein & CoG15 & 5.4 & 0.7 & 0.9 & 2.5 & 1.4 & Nod+ & 1.00 & 1 \\
\hline & RSp0769 & & F4165 & Nonsyn & Putative type VI secretion-associated protein & CoG15 & 6.3 & 1.1 & 1.3 & 1.6 & 1.8 & Nod+ Prot & 17.20 & $3.98 \mathrm{E}-10$ \\
\hline & up $^{116}-$ RSp0780 & & $\mathrm{C} / \mathrm{T}$ & Intergenic & Hypothetical protein & CoG15 & 2.5 & 1.1 & 1.1 & 3 & 1.1 & Nod+ & nd & nd \\
\hline & RSp1422 & & G1298E & Nonsyn & Non-ribosomal peptide synthase & CoG15 & 2 & 1.2 & 1.2 & 2.4 & 1.6 & Nod+ & 18.28 & $2.79 E-03$ \\
\hline & PRALTA_0398 & & W719* & Nonsense & Phosphoenol pyruvate synthase & CoG15 & 2.2 & 0.8 & 0.8 & 1.6 & 1.6 & Nod+ Prot & 151.77 & 0 \\
\hline
\end{tabular}

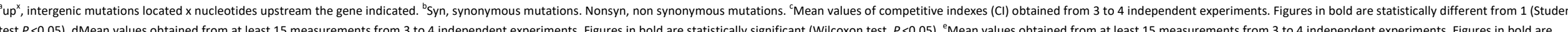
statistically different from 1 (Wilcoxon test, $P<0.05$ with the Benjamini-Hochberg correction). ${ }^{f}$ Rhizot, improvement in rhizosphere colonization. Nodt, improvement in nodulation competitiveness. Pro+t, improvement in within-host proliferation. ${ }^{B} G$ scores were calculated as proposed by Tenaillon et al. (2016). nd, G scores were not determined for mutations in pseudogenes or intergenic regions. ${ }^{h}$ P-values associated with $G$ scores were adjusted using a Bonferroni correction to evaluate genetic parallelism. 